\title{
ON COVARIANT DERIVATIVES AND THEIR APPLICATIONS TO IMAGE REGULARIZATION *
}

\author{
THOMAS BATARD AND MARCELO BERTALMÍO †
}

\begin{abstract}
We present a generalization of the Euclidean and Riemannian gradient operators to a vector bundle, a geometric structure generalizing the concept of manifold. One of the key ideas is to replace the standard differentiation of a function by the covariant differentiation of a section. Dealing with covariant derivatives satisfying the property of compatibility with vector bundle metrics, we construct generalizations of existing mathematical models for image regularization that involve the Euclidean gradient operator, namely the linear scale-space and the Rudin-Osher-Fatemi denoising model. For well-chosen covariant derivatives, we show that our denoising model outperforms stateof-the-art variational denoising methods of the same type both in terms of PSNR and Q-index [45].
\end{abstract}

Key words. Denoising, total variation, scale-space, generalized Laplacian, Riemannian manifold, vector bundle.

1. Introduction. We first give a motivation to the use of covariation differentiation for image processing. Then, we focus in a more detailed manner on how covariant differentiation can be incorporated into variational techniques for image regularization.

1.1. Covariant differentiation for image processing. The notion of covariant differentiation is at the core of the differential geometry of fibre bundles [29],[43]. Whereas covariant differentiation is involved in many mathematical models in theoretical physics, as in general relativity and Gauge fields theories [27], it is involved in very few works in image processing: besides previous work of one of the author $[3],[4],[5],[6],[8]$ we may refer to the work of Duits et al. with applications to crossingpreserving smoothing [19] as well as contour enhancement and completion [17],[18].

Most of the techniques used to perform image processing compare, at some stage, intensity values of neighboring pixels by computing their difference, and this operation can be viewed as a discretization of the standard differentiation of functions. As covariant differentiation of sections of a vector bundle is a generalization of standard differentiation of functions, we claim that image processing techniques can be generalized and hopefully improved by considering an image as a section of a vector bundle and replacing the standard differentiation by covariant differentiation. Then, it raises the problem of the choice of the covariant derivative used to compare intensity of image pixels. In previous works of one of the authors, covariant derivatives were used as parameters that can be tuned to obtain some desirable properties on edge detection $[3]$ and anisotropic diffusion [5],[6]. In [8] and in the current paper, we focus on image denoising, and covariant derivatives are used as parameters too.

Georgiev [21] proposed a theoretical interpretation of the concept of covariant differentiation for images. He argues that covariant differentiation can be used to encode properties of the human visual system, like adaptation which makes the intensity difference perceived between two pixels depending of their surroundings. However the existence and the construction of a covariant derivative that would be theoretically consistent with human perception and will be used to outperform the existing image processing techniques are still open, and non trivial, problems.

*This work was supported by European Research Council, Starting Grant ref. 306337. The authors have published a preliminary version of this work in [8].

${ }^{\dagger}$ Department of Information and Communication Technologies, University Pompeu Fabra, Barcelona, Spain(thomas.batard@upf.edu, marcelo.bertalmio@upf.edu). 
Whereas the use of covariant differentiation as a model for comparing neighboring pixels of natural grey-level and color images is not straightforward, there exist some types of fields where it seems more obvious, like flow fields and manifold-valued fields. Indeed, flow fields can be considered as tangent vector fields over a well-chosen manifold, i.e. sections of a tangent bundle, which makes covariant differentiation on tangent bundle be a tool to compare vectors at difference positions. In particular, if the manifold where the flow is defined is a Riemannian manifold, e.g. a surface in $\mathbb{R}^{3}$, then the field can be differentiated through the Levi-Civita covariant derivative (see an application in [4]). One can also envisage to process manifold-valued fields through the fields (not necessarily unique) of their infinitesimal generators, which take values in the tangent bundle of the manifold, and make use of covariant differentiation on the tangent bundle. This technique was used in [4] for regularizing $S^{1}$-valued fields. We refer to the recent work of Rosman et al. [39] and references therein for others techniques dedicated to manifold-valued fields regularization.

1.2. A short overview on variational methods for image regularization. Variational techniques have been successfully employed in various image processing tasks, e.g. regularization, contrast enhancement, optimal flow estimation. Regarding the problem of image regularization, the seminal techniques involved norms of the Euclidean gradient operator. More precisely, the L2 norm is related with the linear scale-space (see e.g.[34]) and the L1 norm (also called Total Variation and denoted by $\mathrm{TV}$ ) is used in the Rudin-Osher-Fatemi (ROF) denoising model [41]. However, it is well-known that these techniques provide some undesirable effects. Indeed, the linear scale-space does not preserve the edges of the initial condition and the ROF model tends to produce stair-casing effects, over-smoothing and the numerical scheme in [41] does not converge towards the solution of the variational problem. Since then, many techniques have been developed in order to overcome these issues.

In [14], Chambolle extended the ROF denoising model by extending the TV of differentiable functions to functions of bounded variations (BV), and obtained an algorithm for reaching the unique solution of the original ROF model. We refer to Chambolle et al. [15] for a complete introduction to TV and its applications in image analysis. Since then, the approach of Chambolle has been extended in several ways. Bresson and Chan [11] make use of the Vectorial Total Variation (VTV) introduced in [1] to extend Chambolle's algorithm for color image denoising. Based on geometric measure theory, Goldluecke et al. [25] proposed an alternative extension of $\mathrm{TV}$ to vector-valued functions. Let us also mention that a Total Generalized Variation (TGV) that involves higher order derivatives of the image has been defined by Bredies et al. [10] in order to take more into account the local geometry of the image in the denoising process.

Many variational techniques for image regularization involve spatial adaptivity in order to take into account the contours and textures of the image to regularize. Some of these techniques make use of the structure tensor of the image (see for instance [40],[42],[44],[46] for anisotropic diffusion and [33],[38] for denoising). In particular, the approaches in [38],[42] are based on a generalization of the gradient operator to Riemannian manifolds. Let us also cite the recent work of Lenzen et al. [32] who introduced spatial adaptivity in the TGV for denoising purposes. Another denoising method based on second order derivatives of the image is the approach of Zhu and Chan [47] where the TV in the ROF denoising model is replaced by the absolute value of the Mean Curvature of the image surface.

Some recent techniques perform image denoising through the denoising of a field 
attached to the image. For instance, Lysaker et al. [35] first denoise the directions field of the (Euclidean) gradient of the image, and then obtain the denoised image as an image whose directions field of the gradient match with the smoothed directions. Such an approach motivated the Bregman iterations-based denoising algorithm of Osher et al. [26],[36]. More recently, Bertalmío and Levine [9] adopted a similar approach but dealing with the curvature of the level-lines.

Since the seminal works of Awate and Whitaker [2], and Buades et al. [13], nonlocal techniques for image denoising have been developed and turn out to provide much better results than those of state-of-the-art local methods. A non-local variational approach for denoising has been proposed by Gilboa and Osher [24], defining a nonlocal gradient operator and the corresponding non-local ROF denoising model. A non-local extension of the TV based on measure theory has also been proposed more recently by Jin et al. [28].

1.3. Previous work and contribution. In the aforementioned denoising methods that use spatial adaptivity or a field attached to the image, the data involved were directions and strengths of edges, curvature of level-lines, or Gaussian curvature of the image surface. In order to be able to carry more information, we introduced in [8] a generalization of the Euclidean and Riemannian gradient operators. This new gradient operator acts on a section of a vector bundle and is determined by three geometric data: a Riemannian metric on the base manifold, a covariant derivative and a positive definite metric on the vector bundle. In particular, we showed that if the covariant derivative satisfies the property of compatibility with the metric, then the gradient descent flow related with the L2 norm minimization of the gradient operator corresponds to the heat equation of some generalized Laplacian. We also proposed a vector bundle extension of the ROF denoising model based on a regularized L1 norm of such gradients, where the solution can be reached through a gradient descent algorithm. For a well-chosen generalized gradient of color images, we showed that the denoising model outperforms the split Bregman algorithm [22], both in terms of PSNR and Q-index measures [45].

In this paper, we extend the results in [8] in several ways.

1. From a theoretical viewpoint, we establish more results upon heat equations of generalized Laplacians. Moreover, we introduce a Vector Bundle-valued Total Variation (VBTV) that generalizes the TV and VTV to sections of a vector bundle, from which we derive a ROF denoising model without regularization of the L1 norm. We provide the expression of the solutions of this variational problem by showing that the analysis of Chambolle in [14] extends in a straightforward way to a vector bundle provided that the covariant derivative is compatible with the metric. We also introduce an algorithm for reaching the solutions of this denoising model.

2. The generalized gradient we were using in the experiments was determined by an orthonormal moving frame chosen arbitrarily. In this paper, we show empirically that our results are invariant with respect to the choice of the orthonormal moving frame, provided that the moving frame satisfies certain properties.

3. New and extensive quantitative experiments show that the denoising models associated with this approach outperform state-of-the-art variational denoising methods of the same type.

This paper is organized as follows. In Sect. 2, we first review the main properties of covariant derivatives compatible with the metric. Sect. 3 is devoted to the theoretical study of the vector bundle extensions of the linear scale-space and ROF denoising 
model. In particular, we give expressions of the solutions. Finally, in Sect. 4, we provide experiments of our denoising model on both grey-level and color images.

2. On covariant derivatives compatibles with the metric. The main aim of this Section is to show that covariant derivatives that are compatible with the metric share many properties with the Euclidean gradient operator. We also establish a link between the parallel sections associated with such covariant derivatives and harmonic maps between Riemannian manifolds.

\subsection{On the concept of covariant derivatives on a vector bundle.}

2.1.1. Definitions and examples. In this Section, we give the definitions and some examples of the concepts of differential geometry we will use throughout this paper. We refer for instance to [43] for more details on differential geometry of vector bundles.

DEFINITION 2.1. A smooth vector bundle of rank $n$ is a triplet $(E, \pi, M)$ where $M$ and $E$ are two $C^{\infty}$ manifolds, and $\pi: E \longrightarrow M$ is a surjective map such that the preimage $\pi^{-1}(x)$ of $x \in M$ is endowed with a structure of vector space of dimension n. $M$ is called the base manifold and $E$ the total space of the vector bundle. The set $\pi^{-1}(x)$ is called the fiber over $x$, and is denoted by $E_{x}$.

The vector bundle is said to be locally trivial if the following conditions hold: for each $x \in M$, there is a neighborhood $U$ of $x$ and a diffeomorphism $\phi: U \times \mathbb{R}^{n} \longrightarrow$ $\pi^{-1}(U)$ satisfying $\pi \circ \phi(x, f)=x \forall f \in \mathbb{R}^{n}$, and such that the map $\phi_{x}: \mathbb{R}^{n} \longrightarrow E_{x}$ is a vector space isomorphism. The couple $(U, \phi)$ is called a local trivialization.

The vector bundle is said to be trivial if there exists a diffeomorphism $\Phi: M \times$ $\mathbb{R}^{n} \longrightarrow E$ satisfying $\pi \circ \Phi(x, f)=x \forall f \in \mathbb{R}^{n}$, and such that the map $\Phi_{x}: \mathbb{R}^{n} \longrightarrow E_{x}$ is a vector space isomorphism.

Example 1. Let $M$ be a $C^{\infty}$ manifold of dimension $m$. The disjoint union of tangent spaces $T M:=\bigsqcup T_{x} M$, for $x \in M$, is the total space of a vector bundle $(T M, \pi, M)$ of rank $m$ called the tangent bundle of $M$.

Definition 2.2. A metric $h$ on a vector bundle is the assigment of a scalar product $h_{x}$ on each fiber $\pi^{-1}(x)$.

Example 2. A Riemannian metric on a manifold is a positive definite metric on its tangent bundle.

Definition 2.3. A section of a smooth vector bundle $(E, \pi, M)$ is a differentiable map $S: M \longrightarrow E$ such that $\pi \circ S=I d_{M}$.

Let $\left(f_{1}, \cdots, f_{n}\right)$ be a basis of $\mathbb{R}^{n}$. In a local trivialization $(U, \phi)$ of $(E, \pi, M)$, any section may be written

$$
S(x)=\sum_{i=1}^{n} s_{i}(x) \phi\left(x, f_{i}\right)
$$

for some functions $s_{i} \in C^{\infty}(U)$.

The set $\left\{\phi\left(\cdot, f_{1}\right), \cdots, \phi\left(\cdot, f_{n}\right)\right\}$ is called a local frame of $(E, \pi, M)$. 
In this paper, we will denote by $\Gamma(E)$ the set of smooth sections of $(E, \pi, M)$.

Example 3. The tangent vector fields on $M$ are the sections of the tangent bundle $(T M, \pi, M)$.

DEFINITION 2.4. A covariant derivative (or connection) on $(E, \pi, M)$ is a map $\nabla^{E}: \Gamma(T M) \times \Gamma(M) \longrightarrow \Gamma(M)$ satisfying the following axioms:

$-\nabla_{X}^{E}(\varphi+\psi)=\nabla_{X}^{E} \varphi+\nabla_{X}^{E} \psi$

$-\nabla_{X}^{E} f \varphi=d_{X} f \varphi+f \nabla_{X}^{E} \varphi$

$-\nabla_{X}^{E}+Y \varphi=\nabla_{X} \varphi+\nabla_{Y} \varphi$

$-\nabla_{f X}^{E} \varphi=f \nabla_{X}^{E} \varphi$

$\forall X, Y \in \Gamma(T M), f, g \in C^{\infty}(M), \varphi, \psi \in \Gamma(E)$.

It follows from the axioms above that $\nabla^{E}$ can also written as

$$
\nabla_{X}^{E} \varphi=d_{X} \varphi+\omega^{E}(X) \varphi
$$

where $d$ stands for the differential operator acting on the components of the sections and $\omega^{E} \in \Gamma\left(T^{*} M \otimes E n d(E)\right)$ is called a connection 1-form.

Hence, a covariant derivative is completely determined by a connection 1-form.

Example 4 (trivial covariant derivative). The set of smooth functions $C^{\infty}(M)$ on a manifold $M$ can be viewed as a (trivial) vector bundle of rank 1 over $M$. Then, the covariant derivative determined by the connection 1-form $\omega^{E} \equiv 0$ corresponds to the standard differential operator $d$, that is also called trivial covariant derivative.

Example 5 (Levi-Civita connection). Let $(M, g)$ be a Riemannian manifold. The Levi-Civita connection is the covariant derivative on its tangent bundle defined by the following connection 1-form

$$
\omega_{j k}^{E}\left(\partial / \partial x_{i}\right):=\frac{1}{2} g^{k l}\left(\partial_{j} g_{l i}+\partial_{i} g_{l j}-\partial_{l} g_{i j}\right), \quad i, j, k=1, \cdots, \operatorname{dim} M
$$

2.1.2. Covariant derivative and gradient operator. The duality between the trivial covariant derivative and the gradient operator of functions on a Riemannian manifold (and Euclidean space in particular) can be used to construct a new class of regularizers based on covariant derivatives.

The vector bundle $T^{*} M$ of differential 1 -forms on a Riemannian manifold $(M, g)$ of dimension $m$ is naturally equipped with the Riemannian metric $g^{-1}$. The musical isomorphism $\sharp: T^{*} M \longmapsto T M$ maps $d f$ onto the Riemannian gradient $\nabla f$ of $f$, of components $g^{i j} \partial f / \partial x_{i}$ in the frame $\left\{\partial / \partial x_{j}\right\}$. Moreover the norm of $d f$ coincides with the norm of $\nabla f$ since we have

$$
\|d f\|_{g^{-1}}:=\sqrt{\langle d f, d f\rangle_{g^{-1}}}=\sqrt{\sum_{i, j=1}^{m} g^{i j} \frac{\partial f}{\partial x_{i}} \frac{\partial f}{\partial x_{j}}}=\|\nabla f\|_{g}
$$


As a consequence, the standard regularizers of the form

$$
\int_{M} \Phi\left(\|\nabla \varphi\|_{g}^{p}\right) d M
$$

for $\varphi \in C^{\infty}(M), \Phi: \mathbb{R} \longrightarrow \mathbb{R}^{+}$and $p>0$, can be replaced by

$$
\int_{M} \Phi\left(\|d \varphi\|_{g^{-1}}^{p}\right) d M
$$

Let $E$ be a vector bundle over a Riemannian manifold $(M, g)$ of dimension $m$ equipped with a covariant derivative $\nabla^{E}$ and a positive definite metric $h$. The metrics $g$ on $T M$ and $h$ on $E$ induce the positive definite metric $g^{-1} \otimes h$ on $T^{*} M \otimes E$. In particular, for $\varphi, \psi \in \Gamma(E)$, we have

$$
\left\langle\nabla^{E} \varphi, \nabla^{E} \psi\right\rangle_{g^{-1} \otimes h}=\sum_{i, j=1}^{m} g^{i j}\left\langle\nabla_{\partial / \partial x_{i}}^{E} \varphi, \nabla_{\partial / \partial x_{j}}^{E} \psi\right\rangle_{h}
$$

This leads to a generalization of the standard regularizers (2.3) given by

$$
\int_{M} \Phi\left(\left\|\nabla^{E} \varphi\right\|_{g^{-1} \otimes h}^{p}\right) d M
$$

2.2. Properties of covariant derivatives compatibles with the metric.

2.2.1. Definition and examples. Let $E$ be a vector bundle over a Riemannian manifold $(M, g)$ equipped with a positive definite metric $h$.

Definition 2.5. A covariant derivative $\nabla^{E}$ on $E$ is compatible with the metric $h$ if it satisfies

$$
d h(\varphi, \psi)=h\left(\nabla^{E} \varphi, \psi\right)+h\left(\varphi, \nabla^{E} \psi\right)
$$

for any $\varphi, \psi \in \Gamma(E)$.

Example 6. Assuming that $E$ is the vector bundle of smooth functions on $(M, g)$ and $h$ is nothing but the scalar multiplication in the fibers, the trivial covariant derivative $d$ is compatible with the metric $h$.

Example 7. Assuming that $E$ is the tangent bundle of $(M, g)$, the Levi-Civita connection on $E$ is compatible with the Riemannian metric $g$.

Proposition 2.6. There is a one-one correspondence between connection 1forms $\omega^{E}$ that are $\mathfrak{s o}(h)$-valued i.e. $\omega^{E} \in \Gamma\left(T^{*} M \otimes \mathfrak{s o}(h)\right)$ and covariant derivatives $\nabla^{E}$ that are compatible with the metric.

We refer to Lawson and Michelson [19, Prop. 4.4 p.103] for a proof.

2.2.2. Adjoint and Laplacian operators. Let $\nabla^{T^{*} M \otimes E}$ be the covariant derivative on $T^{*} M \otimes E$ defined by

$$
\nabla^{T^{*} M \otimes E}(\xi \otimes \varphi)=\nabla^{T^{*} M} \xi \otimes \varphi+\xi \otimes \nabla^{E} \varphi
$$


where $\nabla^{T^{*} M}$ is the covariant derivative on $T^{*} M$ induced by the Levi-Civita covariant derivative on $(T M, g)$ and $\nabla^{E}$ is a covariant derivative on $E$ compatible with a positive definite metric $h$. The adjoint $\nabla^{E^{*}}: \Gamma\left(T^{*} M \otimes E\right) \longrightarrow \Gamma(E)$ of the operator $\nabla^{E}: \Gamma(E) \longrightarrow \Gamma\left(T^{*} M \otimes E\right)$ is the operator

$$
\nabla^{E^{*}}=-\operatorname{Tr} \nabla^{T^{*} M \otimes E}
$$

where $\operatorname{Tr}$ denotes the contraction with respect to the metric $g$. In others words, the following equality is satisfied

$$
\int_{M}\left\langle\varphi, \nabla^{E^{*}} \eta\right\rangle_{h} d M=\int_{M}\left\langle\nabla^{E} \varphi, \eta\right\rangle_{g^{-1} \otimes h} d M
$$

assuming that one of $\varphi$ or $\eta$ has compact support.

Example 8. On the vector bundle of smooth functions on a Riemannian manifold $(M, g)$, the adjoint $d^{*}: \Gamma\left(T^{*} M\right) \longrightarrow C^{\infty}(M)$ of the trivial covariant derivative $d: C^{\infty}(M) \longrightarrow \Gamma\left(T^{*} M\right)$ is the operator

$$
d^{*} \eta=-\sum_{i, j}\left(g^{i j} \partial_{x_{i}} \eta\left(\partial / \partial x_{j}\right)-\sum_{k} \Gamma_{i j}^{k} \eta\left(\partial / \partial x_{k}\right)\right)
$$

where $\Gamma_{i j}^{k}$ are the Christoffel symbols of the Levi-Civita connection of $(M, g)$.

Assuming that $g$ is Euclidean and under the identification between 1-forms and vector fields, we obtain the divergence operator (up to a sign).

DEFINITION 2.7. The connection Laplacian $\Delta^{E}$ associated to the covariant derivative $\nabla^{E}$ is the second order differential operator defined by $\Delta^{E}=\nabla^{E^{*}} \nabla^{E}$.

In the frame $\left(\partial / \partial x_{1}, \cdots, \partial / \partial x_{m}\right)$ of $(T M, g)$, we have

$$
\Delta^{E}=-\sum_{i j} g^{i j}\left(\nabla_{\partial / \partial x_{i}}^{E} \nabla_{\partial / \partial x_{j}}^{E}-\sum_{k} \Gamma_{i j}^{k} \nabla_{\partial / \partial x_{k}}^{E}\right)
$$

Example 9. The Laplace-Beltrami operator $\Delta_{g}$ is the connection Laplacian (up to a sign) associated to the trivial covariant derivative $d$ on the vector bundle of smooth functions on a Riemannian manifold $(M, g)$, i.e.

$$
\Delta_{g}=\sum_{i j} g^{i j}\left(\partial_{x_{i}} \partial_{x_{j}}-\sum_{k} \Gamma_{i j}^{k} \partial_{x_{k}}\right)
$$

2.2.3. On the corresponding parallel sections. The aim of this Section is to establish a relation between the geometric framework we introduce in this paper and the Beltrami framework of Sochen et al. [42] which deals with harmonic mapping between Riemannian manifolds. 
Construction of parallel sections. Given a covariant derivative $\nabla^{E}$ (not necessarily compatible with the metric) on a vector bundle $E$, the sections $\varphi$ satisfying $\nabla^{E} \varphi \equiv 0$ are called parallel.

In the following Proposition, we show that, under the assumption that the covariant derivative is compatible with the metric, parallel sections can be constructed from heat equations of connection Laplacians.

Proposition 2.8. Let $E$ be a vector bundle over a compact Riemannian manifold $(M, g)$ equipped with a positive definite metric $h$ and a covariant derivative $\nabla^{E}$ compatible with $h$. The gradient descent algorithm for reaching a solution of the variational problem

$$
\underset{\varphi \in \Gamma(E)}{\arg \min } J(\varphi):=\int_{M}\left\|\nabla^{E} \varphi\right\|_{g^{-1} \otimes h}^{2} d M
$$

is the Euler scheme associated to the (generalized) heat equation

$$
\frac{\partial \varphi}{\partial t}+\Delta^{E} \varphi=0
$$

Proof. Let $\psi$ be a smooth section with compact support. We have

$$
\frac{d}{d t} J(\varphi+t \psi)_{\mid t=0}=\int_{M}\left\langle\nabla^{E} \varphi, \nabla^{E} \psi\right\rangle_{g^{-1} \otimes h} d M
$$

Then, as $\nabla^{E}$ is compatible with the metric, we have

$$
\int_{M}\left\langle\nabla^{E} \varphi, \nabla^{E} \psi\right\rangle_{g^{-1} \otimes h} d M=\int_{M}\left\langle\Delta^{E} \varphi, \psi\right\rangle_{h} d M
$$

See for instance [19, Prop. 8.1 p.154] for a proof of (2.12). Hence, we have

$$
\frac{d}{d t} J(\varphi+t \psi)_{\mid t=0}=0 \Longrightarrow \int_{M}\left\langle\Delta^{E} \varphi, \psi\right\rangle_{h} d M=0
$$

Finally, from a straightforward generalization of the Euclidean case

$$
\int_{M} \Delta f g d M=0 \Longrightarrow \Delta f \equiv 0
$$

where $\Delta$ is the Euclidean Laplacian, $f \in C^{\infty}(M)$ and $g \in C_{c}^{\infty}(M)$, we deduce that

$$
\frac{d}{d t} J(\varphi+t \psi)_{\mid t=0}=0 \Longrightarrow \Delta^{E} \varphi \equiv 0
$$

Finally, as the functional $J$ is convex, the gradient descent associated to the variational problem (2.10)

$$
\varphi_{t+d t}=\varphi_{t}-d t \Delta^{E} \varphi_{t}
$$

converges towards a global minimum.

As the parallel sections are the solutions of the variational problem (2.10), we deduce that the solutions of the heat equation (2.11) converge towards parallel sections when $t \rightarrow+\infty$. 
Parallel sections and harmonic maps. Let $(M, g)$ and $(N, Q)$ be two smooth Riemannian manifolds. The Dirichlet energy of a map $\sigma:(M, g) \longrightarrow(N, Q)$ is defined by

$$
E(\sigma)=\int_{M}\|d \sigma\|_{g^{-1} \otimes Q}^{2} d M
$$

The critical points of (2.13) are called harmonic maps.

Definition 2.9 (Sasaki metric). Let $E$ be a vector bundle over a compact Riemannian manifold $(M, g)$ equipped with a metric $h$ and a covariant derivative $\nabla^{E}$ compatible with $h$. Let ${ }^{H}: T M \longrightarrow T E$ and ${ }^{V}: E \longrightarrow T E$ be respectively the horizontal and vertical lift maps induced by $\nabla^{E}$, and $K: T E \longrightarrow E$ be the map defined by

$$
K\left(X^{H}\right)=0 \quad K\left(\varphi^{V}\right)=\varphi, \quad \forall X \in \Gamma(T M), \varphi \in \Gamma(E)
$$

The Sasaki metric $\tilde{h}$ is the Riemannian metric on $E$ defined by

$$
\tilde{h}(X, Y)=g\left(\pi_{*} X, \pi_{*} Y\right)+h(K X, K Y)
$$

Taking the Sasaki metric $\tilde{h}$ on $E$ induced $\left(h, \nabla^{E}, g\right)$, the Dirichlet energy (2.13) of $\varphi \in \Gamma(E)$, seen as a map $\varphi:(M, g) \longrightarrow(E, \tilde{h})$, is

$$
E(\varphi)=\frac{n}{2} \operatorname{Vol}(M)+\frac{1}{2} \int_{M}\left\|\nabla^{E} \varphi\right\|_{g^{-1} \otimes h}^{2} d M
$$

It follows the theorem that relates harmonic maps and parallel sections

THEOREM 2.10 (Konderak [30]). Let $E$ be a vector bundle over a compact Riemannian manifold $(M, g)$ equipped with a metric $h$ and a covariant derivative $\nabla^{E}$ compatible with $h$. Let $\tilde{h}$ be the Sasaki metric on $E$ associated to $\left(h, \nabla^{E}, g\right)$. Then $\varphi \in \Gamma(E)$ is a harmonic map $\varphi:(M, g) \longrightarrow(E, \tilde{h})$ if and only if it is parallel, i.e. $\nabla^{E} \varphi \equiv 0$.

3. Vector bundle extension of image regularization methods. From the properties of a covariant derivative compatible with the metric given in Sect. 2, we show in this Section that the linear scale-space and the ROF denoising model, which are standard image regularization techniques, can be generalized in straightforward way by replacing the Euclidean gradient operator by a covariant derivative compatible with the metric.

3.1. Functional spaces on vector bundles. Let $E$ be a smooth vector bundle over a compact Riemannian manifold $(M, g)$, equipped with a positive definite metric $h$ and a covariant derivative $\nabla^{E}$. Let \|\|$_{k}^{p}$ be the norm defined for $\varphi \in \Gamma(E)$ by

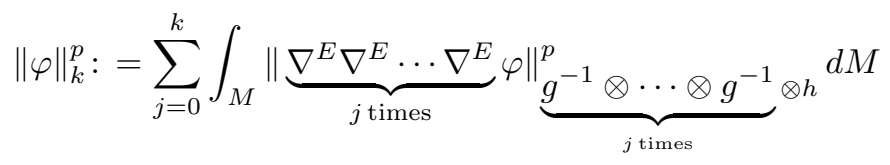

The equivalence class of this norm is independent of the choice of the connection $\nabla^{E}$ and metrics $g, h$. 
Definition 3.1. The completion of $\Gamma(E)$ in this norm is the Sobolev space $W^{k, p}(E)$. We denote by $L^{p}(E)$ the spaces $W^{0, p}(E)$.

Definition 3.2. The Vector Bundle-valued Total Variation (VBTV) of $\varphi \in L_{l o c}^{1}(E)$ is

$V B T V(\varphi):=\sup \left(\int_{M}\left\langle\varphi, \nabla^{E^{*}} \eta\right\rangle_{h} d M ; \eta \in \Gamma_{c}\left(T^{*} M \otimes E\right),\|\eta(x)\|_{g^{-1} \otimes h} \leq 1 \quad \forall x \in M\right)$

Definition 3.3 (Bounded variations section). A section $\varphi \in L_{\text {loc }}^{1}(E)$ is said to have a Bounded Variation if $V B T V(\varphi)<+\infty$. We denote by $B V(E)$ the space of sections having a bounded variation.

Proposition 3.4. Let us assume that $\nabla^{E}$ is compatible with $h$. If $\varphi \in W^{1,1}(E)$, we have

$$
\operatorname{VBTV}(\varphi)=\int_{M}\left\|\nabla^{E} \varphi\right\|_{g^{-1} \otimes h} d M
$$

Proof. As $\nabla^{E}$ is compatible with the metric and $\eta \in \Gamma_{c}\left(T^{*} M \otimes E\right)$, we have

$$
\int_{M}\left\langle\varphi, \nabla^{E^{*}} \eta\right\rangle_{h} d M=\int_{M}\left\langle\nabla^{E} \varphi, \eta\right\rangle_{g^{-1} \otimes h} d M
$$

See for instance [19, Prop. 8.1 p.154] for a proof of the equality.

Then, as the metric $g^{-1} \otimes h$ is positive definite,

$$
\int_{M}\left\langle\nabla^{E} \varphi, \eta\right\rangle_{g^{-1} \otimes h} d M \leq \int_{M}\left\|\nabla^{E} \varphi\right\|_{g^{-1} \otimes h}\|\eta\|_{g^{-1} \otimes h} d M
$$

Moreover, we get

$$
\int_{M}\left\|\nabla^{E} \varphi\right\|_{g^{-1} \otimes h}\|\eta\|_{g^{-1} \otimes h} d M \leq \int_{M}\left\|\nabla^{E} \varphi\right\|_{g^{-1} \otimes h} d M
$$

since $\|\eta\|_{g^{-1} \otimes h} \leq 1 \forall x \in M$. Hence

$\sup \left(\int_{M}\left\langle\varphi, \nabla^{E^{*}} \eta\right\rangle_{h} d M ; \eta \in \Gamma_{c}\left(T^{*} M \otimes E\right),\|\eta(x)\|_{g^{-1} \otimes h} \leq 1 \quad \forall x \in M\right) \leq \int_{M}\left\|\nabla^{E} \varphi\right\|_{g^{-1} \otimes h} d M$

Let

$$
\tilde{\eta}:=\left\{\begin{array}{cc}
\frac{\nabla^{E} \varphi}{\left\|\nabla^{E} \varphi\right\|_{g^{-1} \otimes h}} & \text { if } \nabla^{E} \varphi \neq 0 \\
0 & \text { otherwise }
\end{array}\right.
$$

and $\left(\eta_{\epsilon}\right) \in \Gamma_{c}^{1}\left(T^{*} M \otimes E\right),\left\|\eta_{\epsilon}(x)\right\|_{g^{-1} \otimes h} \leq 1 \quad \forall x \in M$ such that $\eta_{\epsilon} \underset{\epsilon \rightarrow 0}{\longrightarrow} \tilde{\eta}$. We claim that such a sequence can be constructed using generalizations of Lusin's theorem and the mollification technique to vector-valued measurable functions.

Then we have

$$
\lim _{\epsilon \rightarrow 0} \int_{M}\left\langle\varphi, \nabla^{E^{*}} \eta_{\epsilon}\right\rangle_{h} d M=\lim _{\epsilon \rightarrow 0} \int_{M}\left\langle\nabla^{E} \varphi, \eta_{\epsilon}\right\rangle_{g^{-1} \otimes h} d M=\int_{M}\left\langle\nabla^{E} \varphi, \tilde{\eta}\right\rangle_{g^{-1} \otimes h} d M
$$




$$
=\int_{\left\{\nabla^{E} \varphi \neq 0\right\}}\left\langle\nabla^{E} \varphi, \frac{\nabla^{E} \varphi}{\left\|\nabla^{E} \varphi\right\|_{g^{-1} \otimes h}}\right\rangle_{g^{-1} \otimes h} d M=\int_{M}\left\|\nabla^{E} \varphi\right\|_{g^{-1} \otimes h} d M
$$

Hence, we have constructed a sequence $\int_{M}\left\langle\varphi, \nabla^{E^{*}} \eta_{\epsilon}\right\rangle_{h} d M$ converging towards $\int_{M}\left\|\nabla^{E} \varphi\right\|_{g^{-1} \otimes h} d M$. Moreover

$$
\int_{M}\left\langle\varphi, \nabla^{E^{*}} \eta_{\epsilon}\right\rangle_{h} d M \leq \int_{M}\left\|\nabla^{E} \varphi\right\|_{g^{-1} \otimes h} d M \quad \forall \epsilon
$$

since $\left\|\eta_{\epsilon}(x)\right\|_{g^{-1} \otimes h} \leq 1 \quad \forall x \in M$. Together with (3.4), it shows that

$\sup \left(\int_{M}\left\langle\varphi, \nabla^{E^{*}} \eta\right\rangle_{h} d M ; \eta \in \Gamma_{c}\left(T^{*} M \otimes E\right),\|\eta(x)\|_{g^{-1} \otimes h} \leq 1 \forall x \in M\right)=\int_{M}\left\|\nabla^{E} \varphi\right\|_{g^{-1} \otimes h} d M$

$\square$

3.2. Scale-space on vector bundles. In this Section, we show that some properties of the Euclidean scale-space extend in a straightforward way to vector bundles.

Let us first note that a differential operator $H: \Gamma(E) \longrightarrow \Gamma(E)$ of order $m$ extends to a bounded linear map $H: W_{k}^{p} \longrightarrow W_{k-m}^{p}$ for all $k \geq m$.

Proposition 3.5. Let $E$ be a vector bundle over a compact Riemannian manifold $(M, g)$ equipped with a positive definite metric $h$ and a covariant derivative $\nabla^{E}$ compatible with $h$. The solution $e^{-t \Delta^{E}} \varphi_{0}$ of the heat equation

$$
\frac{\partial \varphi}{\partial t}+\Delta^{E} \varphi=0, \quad \varphi_{\mid t=0}=\varphi_{0}
$$

is

$$
e^{-t \Delta^{E}} \varphi_{0}=\sum_{k} e^{-\lambda_{k} t} P_{E_{\lambda_{k}}} \varphi_{0}
$$

where $P$ denotes the L2 projection operator and $E_{\lambda_{k}}$ is the space of eigensections of $\Delta^{E}$ for its kth smallest eigenvalue $\lambda_{k}$.

Proof. If the covariant derivative $\nabla^{E}$ is compatible with the metric of the vector bundle, the elliptic operator $\Delta^{E}$ is positive and self-adjoint. As a consequence, the eigenvalues $\lambda_{k}$ of $\Delta^{E}$ are real, the corresponding eigenspaces $E_{\lambda_{k}}$ are finitedimensional and furnish a complete orthonormal system for $L^{2}(E)$, i.e. there is a Hilbert space direct sum decomposition

$$
L^{2}(E)=\oplus_{\lambda_{k}} E_{\lambda_{k}}
$$

(see [19, Cor.5.9 p.198]). Given a complete orthonormal basis $\left\{u_{k j}\right\}_{k=1}^{\infty}$ of $L^{2}(E)$ consisting of eigensections of $\Delta^{E}$ with $\Delta^{E} u_{k j}=\lambda_{k} u_{k j}$, it is easy to see that the solution of (3.6) is given by

$$
e^{-t \Delta^{E}} \varphi_{0}(x)=\int_{M} K_{t}(x, y) \varphi_{0}(y) d y
$$

where $K_{t}$ is called the heat kernel of the connection Laplacian $\Delta^{E}$ and is given by

$$
K_{t}(x, y)=\sum_{k} \sum_{j=1}^{\operatorname{dim} E_{k}} e^{-\lambda_{k} t} u_{k j}(x) \otimes u_{k j}^{*}(y)
$$


where $v^{*} \in E_{y}^{*}$ denotes the element such that $v^{*}(u)=\langle u, v\rangle_{h_{y}}$ for all $u \in E_{y}$.

Hence

$$
\begin{aligned}
e^{-t \Delta^{E}} \varphi_{0}(x) & =\sum_{k} e^{-\lambda_{k} t} \int_{M} \sum_{j=1}^{\operatorname{dim} E_{\lambda_{k}}} u_{k j}(x) \otimes u_{k j}{ }^{*}(y) \varphi_{0}(y) d y \\
& =\sum_{k} e^{-\lambda_{k} t} \int_{M} \sum_{j=1}^{\operatorname{dim} E_{\lambda_{k}}}\left\langle\varphi_{0}(y), u_{k j}(y)\right\rangle_{h_{y}} u_{k j}(x) d y \\
& =\sum_{k} e^{-\lambda_{k} t} \sum_{j=1}^{\operatorname{dim} E_{k}}\left\langle\varphi_{0}, u_{k j}\right\rangle_{L^{2}(E)} u_{k j}(x) \\
& =\sum_{k} e^{-\lambda_{k} t} P_{E_{\lambda_{k}}} \varphi_{0}(x)
\end{aligned}
$$

$\square$

Note that we showed in Sect. 2.2.3 that the sequence $\left(e^{-t \Delta^{E}} \varphi_{0}\right)_{t \geq 0}$ converges towards a parallel section.

The following result shows that heat equations of connections Laplacians where the covariant derivative is compatible with the metric satisfy some kind of maximum principle.

Corollary 3.6. The sequence $\left\|e^{-t \Delta^{E}} \varphi_{0}\right\|_{L^{2}(E)}, t \geq 0$ is strictly decreasing.

Proof.

$$
\begin{gathered}
\left\|e^{-t \Delta^{E}} \varphi_{0}\right\|_{L^{2}(E)}:=\sqrt{\left\langle e^{-t \Delta^{E}} \varphi_{0}, e^{-t \Delta^{E}} \varphi_{0}\right\rangle_{L^{2}(E)}} \\
=\sqrt{\left\langle\sum_{k} \sum_{j=1}^{\operatorname{dim} E_{\lambda_{k}}} e^{-\lambda_{k} t}\left\langle\varphi_{0}, u_{k j}\right\rangle_{L^{2}(E)} u_{k j}, \sum_{i} \sum_{l=1}^{\operatorname{dim} E_{\lambda_{i}}} e^{-\lambda_{i} t}\left\langle\varphi_{0}, u_{i l}\right\rangle_{L^{2}(E)} u_{i l}\right\rangle_{L^{2}(E)}}
\end{gathered}
$$

Then, as the $\left\{u_{k j}\right\}$ and $\left\{u_{i l}\right\}$ form orthonormal systems, we deduce that

$$
\left\|e^{-t \Delta^{E}} \varphi_{0}\right\|_{L^{2}(E)}=\sqrt{\sum_{k} \sum_{j=1}^{\operatorname{dim} E_{k}} e^{-2 \lambda_{k} t}\left\langle\varphi_{0}, u_{k j}\right\rangle_{L^{2}(E)}^{2}}
$$

which is clearly strictly decreasing. $\square$ 
3.3. ROF denoising model on vector bundles. Let $E$ be a vector bundle over a compact Riemannian manifold $(M, g)$ equipped with a positive definite metric $h$ and a covariant derivative $\nabla^{E}$ compatible with $h$.

Proposition 3.7. Let $f \in L^{2}(E)$ and $\lambda \in \mathbb{R}$. The unique solution of

$$
\underset{\varphi \in B V \cap L^{2}(E)}{\arg \min } \frac{1}{2 \lambda}\|\varphi-f\|_{L^{2}(E)}+\operatorname{VBTV}(\varphi)
$$

is defined by

$$
\varphi=f-P_{\lambda K} f
$$

where $P_{\lambda K}$ denotes the orthogonal projection on $\lambda K$ for $K$ being the closure of the set

$$
\left\{\nabla^{E^{*}} \eta ; \eta \in \Gamma_{c}\left(T^{*} M \otimes E\right),\|\eta(x)\|_{g^{-1} \otimes h} \leq 1 \quad \forall x \in M\right\}
$$

Moreover, if we define the iterative sequence

$$
\xi_{n+1}=\frac{\xi_{n}+\tau \nabla^{E}\left(\nabla^{E^{*}} \xi_{n}-f / \lambda\right)}{1+\tau\left\|\nabla^{E}\left(\nabla^{E^{*}} \xi_{n}-f / \lambda\right)\right\|_{g^{-1} \otimes h}}
$$

then the sequence $\lambda \nabla^{E^{*}} \xi^{n}$ converges to $P_{\lambda K}(f)$ as $n \rightarrow \infty$ assuming that the time step $\tau \leq \frac{1}{\left\|\nabla^{E *}\right\|}$.

Proof. The proof is straightforward since the Proposition is a particular case of the Generalized Projection Algorithm of Chambolle (see e.g. [8, Th. 3.4] for more details).

3.4. Geometric triplets that lead to existing regularization methods. The scale-space and denoising model on vector bundles we have presented in the previous Sections are completely determined by three geometric data:

- a positive definite metric $h$ on a vector bundle $(E, \pi, M)$

- a covariant derivative $\nabla^{E}$ on $E$ compatible with $h$

- a Riemannian metric $g$ on the base manifold $M$.

In this Section, we show that we obtain some existing regularization methods for some particular choice of $h, \nabla^{E}, g$.

3.4.1. Batard and Sochen's model. Let $E$ be a trivial vector bundle of rank 3 over the domain $\Omega \subset \mathbb{R}^{2}$ of a color image $I=\left(I^{1}, I^{2}, I^{3}\right)$, and equipped with the metric $h$ acting as the Euclidean scalar product in the fibers. According to Prop. 2.6. in Sect. 2.2.1, a covariant derivative compatible with the metric $h$ is determined by a connection 1-form $\omega^{E} \in \Gamma\left(T^{*} \Omega \times \mathfrak{s o}(3)\right)$, where $\mathfrak{s o}(3)$ is the Lie algebra of $\mathrm{SO}(3)$ i.e. the set of $3 \times 3$ skew-symmetric matrices. In [6], Batard and Sochen constructed an optimal covariant derivative compatible with $h$ as solution of a minimization problem. The corresponding connection 1 -form $\omega^{E}$ is determined by the matrix coefficients

$\omega_{12}^{E}=-\sum_{k=1}^{2} \lambda \kappa\left((b e-d c)\left(I_{x_{l}}^{2} I^{3}-I_{x_{l}}^{3} I^{2}\right)+(b c-a e)\left(I_{x_{l}}^{1} I^{3}-I_{x_{l}}^{3} I^{1}\right)+\left(a d-b^{2}\right)\left(I_{x_{l}}^{1} I^{2}-I_{x_{l}}^{2} I^{1}\right)\right) d x_{l}$ 
$\omega_{13}^{E}=-\sum_{k=1}^{2} \lambda \kappa\left((c e-f b)\left(I_{x_{l}}^{2} I^{3}-I_{x_{l}}^{3} I^{2}\right)+\left(a f-c^{2}\right)\left(I_{x_{l}}^{1} I^{3}-I_{x_{l}}^{3} I^{1}\right)+(b c-a e)\left(I_{x_{l}}^{1} I^{2}-I_{x_{l}}^{2} I^{1}\right)\right) d x_{l}$

$\omega_{23}^{E}=-\sum_{k=1}^{2} \lambda \kappa\left(\left(f d-e^{2}\right)\left(I_{x_{l}}^{2} I^{3}-I_{x_{l}}^{3} I^{2}\right)+(c e-f b)\left(I_{x_{l}}^{1} I^{3}-I_{x_{l}}^{3} I^{1}\right)+(b e-d c)\left(I_{x_{l}}^{1} I^{2}-I_{x_{l}}^{2} I^{1}\right)\right) d x_{l}$

where

$$
\begin{gathered}
a=\delta+\kappa\left[\left(I^{2}\right)^{2}+\left(I^{3}\right)^{2}\right] \quad b=\kappa I^{1} I^{2} \quad c=-\kappa I^{1} I^{3} \\
d=\delta+\kappa\left[\left(I^{1}\right)^{2}+\left(I^{3}\right)^{2}\right] \quad e=\kappa I^{2} I^{3} \quad f=\delta+\kappa\left[\left(I^{1}\right)^{2}+\left(I^{2}\right)^{2}\right]
\end{gathered}
$$

and

$$
\lambda=\frac{1}{2 c b e+f a d-a e^{2}-f b^{2}-c^{2} d}
$$

for some $\kappa, \delta>0$.

Then, taking $g$ as

$$
g=\left(\begin{array}{cc}
1+\kappa \sum_{k=1}^{3}\left(I_{x_{1}}^{k}\right)^{2} & \kappa \sum_{k=1}^{3} I_{x_{1}}^{k} I_{x_{2}}^{k} \\
\kappa \sum_{k=1}^{3} I_{x_{1}}^{k} I_{x_{2}}^{k} & 1+\kappa \sum_{k=1}^{3}\left(I_{x_{2}}^{k}\right)^{2}
\end{array}\right)
$$

in the frame $\left(\partial / \partial x_{1}, \partial / \partial x_{2}\right)$, they considered the heat equation of the corresponding connection Laplacian $\Delta^{E}$. They obtained an anisotropic scale-space preserving better the contours of the initial condition than the Beltrami flow [42] associated to the metric (3.11).

3.4.2. Trivial covariant derivative. Let $I_{0}: \Omega \subset \mathbb{R}^{2} \longrightarrow \mathbb{R}^{m}$ be a $m$-channels image. Let $E$ be the vector bundle of $\mathbb{R}^{m}$-valued functions over $\Omega$ equipped with a Riemannian metric $g$. Let us assume that $E$ is equipped with the metric $h$ acting as the Euclidean metric in the fibers and the trivial covariant derivative $d$.

Beltrami framework. Taking $g$ as the $m$-channel extension of the structure tensor (3.11), the heat equation of the subsequent connection Laplacian $\Delta_{g}$

$$
\frac{\partial I}{\partial t}=\Delta_{g} I, \quad I_{\mid t=0}=I_{0}
$$

corresponds to the regularization method in [42].

Moreover, the denoising model

$$
\underset{I \in B V \cap L^{2}(E)}{\arg \min } \int_{\Omega} \frac{1}{2 \lambda}\left\|I-I_{0}\right\|_{2}+\|d I\|_{g^{-1} \otimes \|} \|_{2} d \Omega
$$

where the regularizing term is the $L_{1}$ norm of the gradient associated to this geometric triplet is the denoising model of Rosman et al. [38]. 
Bresson and Chan denoising model. Considering the VBTV associated to this geometric triplet, the denoising model of Bresson and Chan [11] on Riemannian manifolds reads

$$
\underset{I \in B V \cap L^{2}(E)}{\arg \min } \int_{\Omega} \frac{1}{2 \lambda}\left\|I-I_{0}\right\|_{2} d \Omega+V B T V(I)
$$

Moreover, taking $g$ as the Euclidean metric and $m=1$, we obtain the denoising model of Chambolle [14].

4. A new denoising model for multi-channel images. In this Section, we show that, for a well-chosen class of covariant derivatives compatible with the metric, the denoising model described in Sect. 3.3 outperforms state-of-the-art denoising methods of same type. In Sect. 4.1 we remind the reader of the geometric framework introduced in [7] for edge-preserving scale-space, and also used in [8] for denoising. Then, a modification of these approaches is presented in Sect. 4.2. In Sect. 4.3, we show that this new approach can be viewed as a particular case of the ROF model on vector bundle introduced in Sect. 3.3. We discuss in more details the parameters of our approach in Sect. 4.4. Finally, experiments regarding the choice of the parameters and comparisons with other denoising methods are presented in Sect. 4.5.

4.1. The geometric framework. One of the key ideas of our previous approaches for image regularization is to embed an image in a higher dimensional space.

Let $I_{0}=\left(I_{0}^{1}, \cdots, I_{0}^{m}\right)$ be a $m$-channel image defined on a domain $\Omega$ of $\mathbb{R}^{2}$. We construct a surface $S$ embedded in $\left(\mathbb{R}^{m+2},\|\|_{2}\right)$ parametrized by

$$
\psi:\left(x_{1}, x_{2}\right) \longmapsto\left(x_{1}, x_{2}, \mu I_{0}^{1}\left(x_{1}, x_{2}\right), \cdots, \mu I_{0}^{m}\left(x_{1}, x_{2}\right)\right), \quad \mu>0
$$

and equip $\left(\mathbb{R}^{m+2},\|\|_{2}\right)$ with an orthonormal frame $\left(e_{1}, \cdots, e_{m+2}\right)$ such that

$$
\psi\left(x_{1}, x_{2}\right)=x_{1} e_{1}+x_{2} e_{2}+\sum_{k=1}^{m} \mu I_{0}^{k}\left(x_{1}, x_{2}\right) e_{k+2}
$$

Note that for $\mu=1$, the surface $S$ is the graph of the function $I_{0}$.

We construct an orthonormal frame field $\left(Z_{1}, Z_{2}, N_{1}, \cdots, N_{m}\right)$ of $\left(\mathbb{R}^{m+2},\|\|_{2}\right)$ over $\Omega$, where $Z_{1}, Z_{2} \in \Gamma(T S)$, i.e. $Z_{1}, Z_{2}$ are tangent vector fields of the surface $S$. It follows that $N_{1}, \cdots, N_{m}$ are normal to the surface.

Denoting by $P$ the frame change matrix field from $\left(e_{1}, \cdots, e_{m+2}\right)$ to $\left(Z_{1}, Z_{2}, N_{1}, \cdots, N_{m}\right)$, we denote by $J_{0}=\left(J_{0}^{1}, \cdots, J_{0}^{m+2}\right)$ the components of $I_{0}$ in the new frame, i.e. we get

$$
\left(\begin{array}{c}
J_{0}^{1} \\
J_{0}^{2} \\
J_{0}^{3} \\
\vdots \\
J_{0}^{m+2}
\end{array}\right)=P^{-1}\left(\begin{array}{c}
0 \\
0 \\
I_{0}^{1} \\
\vdots \\
I_{0}^{m}
\end{array}\right)
$$

The idea developed in [7],[8] is first to apply a regularization method on $J_{0}$, then to compute the components of the result $J$ in the fixed frame $\left(e_{1}, \cdots, e_{m+2}\right)$ through 
the inverse transform

$$
\left(\begin{array}{c}
I^{1} \\
I^{2} \\
I^{3} \\
\vdots \\
I^{m+2}
\end{array}\right)=P\left(\begin{array}{c}
J^{1} \\
J^{2} \\
J^{3} \\
\vdots \\
J^{m+2}
\end{array}\right)
$$

The final output of the algorithm is the $m$-channel image $\left(I^{3}, \cdots, I^{m+2}\right)$.

In [7], the regularization method performed on the function $J_{0}$ is an Euclidean heat diffusion

$$
\frac{\partial J}{\partial t}=\Delta J, \quad J_{\mid t=0}=J_{0}
$$

and it produces an edge-preserving scale-space of the original image $I_{0}$ for a right choice of the moving frame $\left(Z_{1}, Z_{2}, N_{1}, \cdots, N_{m}\right)$ and scalar $\mu$.

In [8], using the same moving frame as in [7] but different value for $\mu$, we performed a vectorial extension of the ROF denoising model with regularized L1 norm of the gradient operator

$$
\underset{J}{\arg \min } \int_{\Omega} \frac{1}{2 \lambda}\left\|J-J_{0}\right\|_{2}+\sqrt{\|\nabla J\|_{2}^{2}+\beta} \quad d \Omega, \quad 0<\beta<<1
$$

and showed that this denoising model outperforms the Split Bregman method [26] in both PSNR and Q-index [45] measures.

4.2. The proposed algorithm. Our proposal in this paper is to replace the denoising model (4.5) by the use of the (non regularized) L1 norm of the gradient operator. We obtain the variational problem

$$
\underset{J \in B V \cap L^{2}\left(\Omega, \mathbb{R}^{m+2}\right)}{\arg \min } \int_{\Omega} \frac{1}{2 \lambda}\left\|J-J_{0}\right\|_{2} d \Omega+V T V(J)
$$

where $J_{0}$ is defined by formula (4.2) and $V T V$ is the Vectorial Total Variation defined by

$$
V T V(J)=\sup \left(\int_{\Omega}\left\langle J, \nabla^{*} \xi\right\rangle_{2}, \xi \in C_{c}^{\infty}\left(\Omega, \mathbb{R}^{2(m+2)}\right) \text { s.t. }\|\xi(x)\|_{2} \leq 1 \quad \forall x \in \Omega\right)
$$

where $\nabla^{*} \xi=\left(\operatorname{div} \xi_{1}, \cdots, \operatorname{div} \xi_{m}\right)$.

Note that the variational problems of the form (4.6) are the restrictions to functions defined on Euclidean spaces of the denoising model (3.12). Then, according to [11], the solution of (4.6) is given by

$$
J=J_{0}-P_{\lambda K} J_{0}
$$

where $P_{\lambda K}$ is the orthogonal projection onto the convex set $\lambda K, K$ being the closure of the set

$$
\left\{\nabla^{*} \xi: \xi \in C_{c}^{1}\left(\Omega, \mathbb{R}^{2(m+2)}\right),\|\xi(x)\|_{2} \leq 1 \forall x \in \Omega\right\},
$$

and the algorithm

$$
\xi^{n+1}=\frac{\xi^{n}+d t \nabla\left(\nabla^{*} \xi^{n}-J_{0} / \lambda\right)}{1+d t \| \nabla\left(\nabla^{*} \xi^{n}-J_{0} / \lambda \|_{2}\right.}
$$

converges towards $\xi^{\text {opt }}$ such that $\lambda \nabla^{*} \xi^{\text {opt }}=P_{\lambda K} J_{0}$. 
4.3. The corresponding geometric triplet. In this Section we show that the variational problem (4.6) is equivalent to the following denoising model of the original function $I_{0}=\left(0,0, I_{0}^{1}, \cdots, I_{0}^{m}\right)$

$$
\underset{I \in B V \cap L^{2}(E)}{\arg \min } \int_{\Omega} \frac{1}{2 \lambda}\left\|I-I_{0}\right\|_{2} d \Omega+V B T V(I)
$$

for some VBTV (3.2) on a well-chosen vector bundle $E$.

Let $E$ be the vector bundle of $\mathbb{R}^{m+2}$-valued functions over $\Omega$. We deduce from Sect. 4.2 that the geometric triplet leading to our denoising model is given, in the frames $\left(Z_{1}, Z_{2}, N_{1}, \cdots, N_{m}\right)$ of $E$ and $\left(\partial / \partial x_{1}, \partial / \partial x_{2}\right)$ of $T \Omega$, by

- $h$ is the Euclidean metric in the fibers

$-\nabla^{E}$ is the trivial covariant derivative $d$

- $g$ is the Euclidean metric

In the frame $\left(e_{1}, \cdots, e_{m+2}\right)$ of $E$, the expression of $\nabla^{E}$ is modified since the corresponding connection 1 -form $\omega^{E}$ writes

$$
\omega^{E}=P d P^{-1}
$$

The covariant derivative $\nabla^{E}$ is also compatible with the metric $h$ is the frame $\left(e_{1}, \cdots, e_{m+2}\right)$ since $\omega^{E}$ is $\mathfrak{s o}(\mathfrak{n})$-valued according to formula (4.10) and Prop. 2.6. Hence, the geometric triplet leading to our denoising model (4.9) is the following

- $h$ is the Euclidean metric in the fibers

- $\nabla^{E}$ is associated to the connection 1-form (4.10)

- $g$ is the Euclidean metric

Note that the attached data term in (4.9) is invariant under the frame change matrix field $P$. Indeed, since the moving frame $\left(Z_{1}, Z_{2}, N_{1}, \cdots, N_{m}\right)$ is orthonormal, we have

$$
\left\|I-I_{0}\right\|_{2}:=\left\|P J-P J_{0}\right\|_{2}=\left\|J-J_{0}\right\|_{2}
$$

Remark 1. The scale-space (4.4) writes

$$
\frac{\partial I}{\partial t}=\Delta^{E} I, \quad I_{\mid t=0}=I_{0}
$$

in the frames $\left(e_{1}, \cdots, e_{m+2}\right)$ of $E$ and $\left(\partial / \partial x_{1}, \partial / \partial x_{2}\right)$ of $T \Omega$, where $\Delta^{E}$ is the connection Laplacian (2.9) induced by the geometric triplet given above.

4.4. Selection of parameters. According to the construction in Sect. 4.1., the denoising method proposed in this paper is parametrized by a scalar $\mu$ that determines a surface $S$ and an orthonormal moving frame $\left(Z_{1}, Z_{2}, N_{1}, \cdots, N_{m}\right)$ of $\mathbb{R}^{m+2}$ over the image domain $\Omega$ where $\left(Z_{1}, Z_{2}\right)$ is an orthonormal moving frame of $S$. In this Section, we introduce a moving frame $\left(Z_{1}, Z_{2}, N_{1}, \cdots, N_{m}\right)$ that is intuitively more sound than the one used in our previous approaches [7],[8]. We also discuss the role of the scalar $\mu$. 
Previous approaches. In [7],[8], the moving frame $\left(Z_{1}, Z_{2}, N_{1}, \cdots, N_{m}\right)$ was constructed as follows. The vector field $Z_{1}$ chosen was $\psi_{x_{1}} /\left\|\psi_{x_{1}}\right\|_{2}$ (see formula 4.1 for the definition of $\psi$ ) and $Z_{2}$ was consequently the unique orthonormal (up to a sign) of $Z_{1}$ in $T S$. The normal vectors $N_{1}, \cdots, N_{m}$ were then constructed from the GramSchmidt orthonormalization process applied to the vectors $\left(Z_{1}, Z_{2}, e_{3}, \cdots, e_{m+2}\right)$. By its construction, this moving frame tends to privilege the $x_{1}$ direction and it might affect the result of the regularization method. This observation leads us to consider the following moving frame.

Metric frame. The structure tensor is a fundamental tool in image processing and computer vision, and has been employed for many tasks, like edge detection [16], anisotropic diffusion [44], [46] as well as corner detection and optical flow estimation [12].

A straightforward computation shows that the unit eigenvectors fields $\left(e_{1}, e_{2}\right)$ of the first fundamental form $g$ of the surface $S$ defined by

$$
g=\left(\begin{array}{cc}
1+\mu^{2} \sum_{k=1}^{m}\left(I_{x_{1}}^{k}\right)^{2} & \mu^{2} \sum_{k=1}^{m} I_{x_{1}}^{k} I_{x_{2}}^{k} \\
\mu^{2} \sum_{k=1}^{m} I_{x_{1}}^{k} I_{x_{2}}^{k} & 1+\mu^{2} \sum_{k=1}^{m}\left(I_{x_{2}}^{k}\right)^{2}
\end{array}\right)
$$

in the frame $\left(\partial / \partial x_{1}, \partial / \partial x_{2}\right)$ coincide with the unit eigenvectors fields of the structure tensor of the image $\mu I$, these latters indicating the directions of the highest and lowest variations of the image at each point. Then, the couple

$$
\left(Z_{1}, Z_{2}\right):=\left(\frac{d \psi\left(e_{1}\right)}{\left\|d \psi\left(e_{1}\right)\right\|_{2}}, \frac{d \psi\left(e_{2}\right)}{\left\|d \psi\left(e_{2}\right)\right\|_{2}}\right)
$$

forms an orthonormal moving frame of $T S$, and an orthonormal moving frame $\left(Z_{1}, Z_{2}, N_{1}, \cdots, N_{m}\right)$ of $\left(\mathbb{R}^{m+2},\|\|_{2}\right)$ over $\Omega$ can be constructed from the GramSchmidt orthonormalization process applied to the frame $\left(Z_{1}, Z_{2}, e_{3}, \cdots, e_{m+2}\right)$.

Dealing with a grey-level image $I$, the explicit expression of the frame change matrix field $P$ from $\left(e_{1}, e_{2}, e_{3}\right)$ to $\left(Z_{1}, Z_{2}, N\right)$ is

$$
P=\left(\begin{array}{ccc}
\frac{I_{x_{1}}}{\sqrt{\left(I_{x_{1}}^{2}+I_{x_{2}}^{2}\right)\left(1+\mu^{2}\left(I_{x_{1}}^{2}+I_{x_{2}}^{2}\right)\right)}} & \frac{-I_{x_{2}}}{\sqrt{I_{x_{1}}^{2}+I_{x_{2}}^{2}}} & \frac{-\mu I_{x_{1}}}{\sqrt{1+\mu^{2}\left(I_{x_{1}}^{2}+I_{x_{2}}^{2}\right)}} \\
\frac{I_{x_{2}}}{\sqrt{\left(I_{x_{1}}^{2}+I_{x_{2}}^{2}\right)\left(1+\mu^{2}\left(I_{x_{1}}^{2}+I_{x_{2}}^{2}\right)\right)}} & \frac{I_{x_{1}}}{\sqrt{I_{x_{1}}^{2}+I_{x_{2}}^{2}}} & \frac{-\mu I_{x_{2}}}{\sqrt{1+\mu^{2}\left(I_{x_{1}}^{2}+I_{x_{2}}^{2}\right)}} \\
\frac{\mu\left(I_{x_{1}}^{2}+I_{x_{2}}^{2}\right)}{\sqrt{\left(I_{x_{1}}^{2}+I_{x_{2}}^{2}\right)\left(1+\mu^{2}\left(I_{x_{1}}^{2}+I_{x_{2}}^{2}\right)\right)}} & 0 & \frac{1}{\sqrt{1+\mu^{2}\left(I_{x_{1}}^{2}+I_{x_{2}}^{2}\right)}}
\end{array}\right),
$$

Note that $P$ is not defined when $I_{x_{1}}=I_{x_{2}}=0$. In the experiments, we define $P$ as the Identity matrix $\mathbb{I}_{3}$ when $I_{x_{1}}=I_{x_{2}}=0$.

Fig. 4.1 illustrates the moving frame $\left(Z_{1}, Z_{2}, N\right)$ associated to the grey-level image $I$ of size $512 \times 512$ defined by

$$
I(i, j)=255-\sqrt{(i-512)^{2}+(j-512)^{2}} .
$$



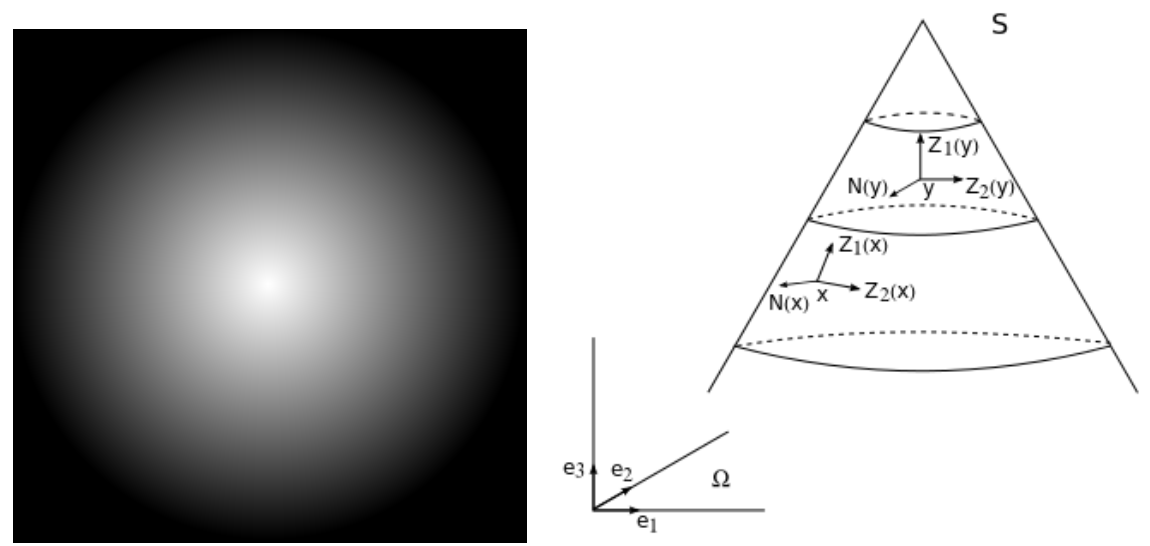

FIG. 4.1. Orthonormal frame field of $\left(\mathbb{R}^{3},\|\|_{2}\right)$ over $\Omega$. Left: Original image. Right: moving frame $\left(Z_{1}, Z_{2}, N\right)$ induced by the metric tensor of the image at two points $x, y$ of $\Omega$.

Fig. 4.1 (left) shows the image $I$, Fig. 4.1 (right) shows the graph $S$ of the function $I$, which is a cone, and how $\left(Z_{1}, Z_{2}, N\right)$ looks like at two points $x$ and $y$ of $\Omega$. As expected, we observe that $Z_{1}$ indicates the directions of the gradient, $Z_{2}$ the directions of the level-lines and $N$ the normal to $S$.

Then, according to the algorithm described above, we apply the denoising model (4.6) to the function $J:=\left(J^{1}, J^{2}, J^{3}\right)$ defined by

$$
\left(\begin{array}{c}
J^{1} \\
J^{2} \\
J^{3}
\end{array}\right):=P^{-1}\left(\begin{array}{l}
0 \\
0 \\
I
\end{array}\right)=\left(\begin{array}{c}
\frac{\mu I\left(I_{x_{1}}^{2}+I_{x_{2}}^{2}\right)}{\sqrt{\left(I_{x_{1}}^{2}+I_{x_{2}}^{2}\right)\left(1+\mu^{2}\left(I_{x_{1}}^{2}+I_{x_{2}}^{2}\right)\right)}} \\
\frac{I}{\sqrt{1+\mu^{2}\left(I_{x_{1}}^{2}+I_{x_{2}}^{2}\right)}}
\end{array}\right)
$$

Fig. 4.2 shows the components $J^{1}$ and $J^{3}$ of the image 'Barbara' for $\mu=0.01$. We observe that the component $J^{1}$ encodes the gradient's norm of the original image $I$, and the component $J^{3}$ appears as the original image to which the gradient's norm has been subtracted, making the components $J^{1}$ and $J^{3}$ be complementary.

The moving frame $\left(Z_{1}, Z_{2}, N_{1}, \cdots N_{m}\right)$. The two orthonormal moving frames aforementioned are both constructed from a Gram-Schmidt procedure applied to the moving frame $\left(Z_{1}, Z_{2}, e_{3}, \cdots, e_{m+2}\right)$, where $\left(Z_{1}, Z_{2}\right)$ forms an orthonormal frame field of the tangent bundle of the surface $S$. Taking $\left(Z_{1}, Z_{2}\right)$ in the tangent bundle of the surface makes the moving frame $\left(Z_{1}, Z_{2}, N_{1}, \cdots N_{m}\right)$ encode the local geometry of the image. Note that we choose the vectors $e_{3}, \cdots, e_{m+2}$ for initializing the Gram-Schmidt process because $Z_{1}, Z_{2}$ (and more generally any tangent vector field to the surface) do not belong to the subspace $\left(e_{3}, \cdots, e_{m+2}\right)$, which makes the Gram-Schmidt procedure be well-defined onto the frame $\left(Z_{1}, Z_{2}, e_{3}, \cdots, e_{m+2}\right)$.

Experiments in Sect. 4.5.1 will show that the proposed denoising method satisfies an invariance property with respect to the choice of the vectors $Z_{1}, Z_{2}$ assuming that they are tangent to the surface. Note also that preliminary tests that we do not present in this paper show that the results are very bad if $Z_{1}, Z_{2}$ are chosen randomly in $\mathbb{R}^{m+2}$. 


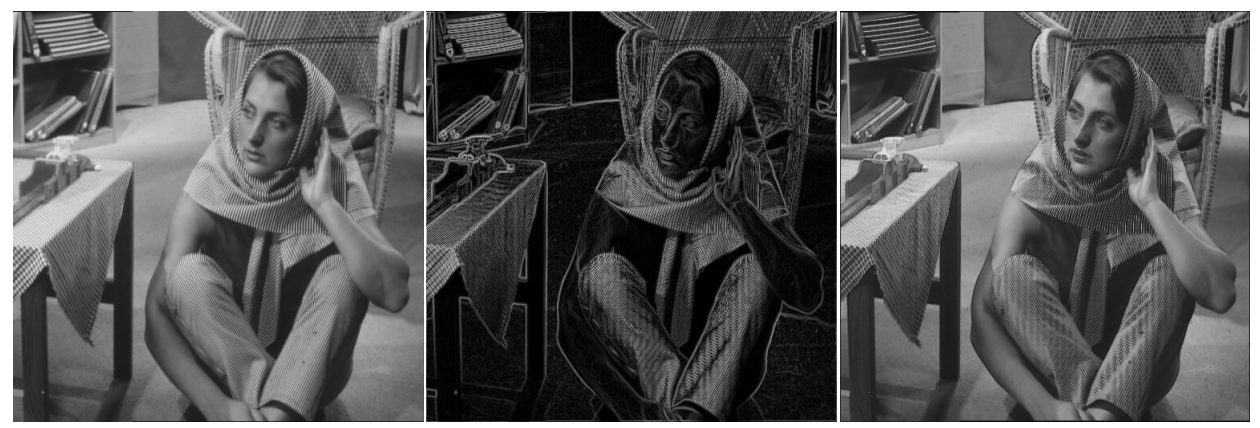

FIG. 4.2. Components of a grey-level image in a moving frame induced by its metric tensor. From left to right: grey-level image "Barbara", component $J^{1}$, component $J^{3}$.

The parameter $\mu$. The scalar $\mu$ plays a fundamental role in our denoising method since it determines the surface $S$ from which we construct the orthonormal moving frame. It is a smoothing parameter for $S$ since it determines the weight of intensities distance with respect to spatial distances (see details in Sect. 4.1). As a consequence, it can be viewed as a smoothing parameter for the moving frame too. Experiments in Sect. 4.5.1 will determine the range of values for $\mu$ that provides the best results for denoising.

For $\mu=0$, our denoising method corresponds to Bresson and Chan's method [11]. Indeed, as mentioned in Sect. 4.2, our denoising method consists in applying Bresson and Chan's algorithm on the components $\left(J_{0}^{1}, \cdots, J_{0}^{m+2}\right)$ of a noisy image $\left(0,0, I_{0}^{1}, \cdots, I_{0}^{m}\right)$ in an orthonormal moving frame. Then, for $\mu=0$, the surface $S$ is nothing but the plane generated by $\left(e_{1}, e_{2}\right)$ and the moving frame $\left(Z_{1}, Z_{2}, N_{1}, \cdots, N_{m}\right)$ is then the fixed frame $\left(e_{1}, \cdots, e_{m+2}\right)$. It follows that the frame change matrix field $P$ is the Identity and the components we denoise are the image itself.

4.5. Experiments. We test our denoising model (4.9) on the Kodak database (http://rok.us/graphics/kodak/) to which we have added Gaussian noise of variance $\sigma$. For the sake of clarity, we remind the reader of the different steps composing our denoising method in the case of a $m$-channel noisy image $I_{0}=\left(I_{0}^{1}, \cdots, I_{0}^{m}\right)$ :

1. Consider the (discrete) surface $S$ parametrized by

$$
\psi:(i, j) \longmapsto\left(i, j, \mu I_{0}^{1}(i, j), \cdots, \mu I_{0}^{m}(i, j)\right), \quad \mu>0
$$

2. Estimate the derivatives of $I_{0}$ using central differences (preliminary tests showed that using others derivative filters does not affect the denoising result in a significant extent). Then, construct an orthonormal moving frame $\left(Z_{1}, Z_{2}, N_{1}, \cdots, N_{m}\right)$ where $Z_{1}, Z_{2}$ are tangent to $S$, and $\left(N_{1}, \cdots, N_{m}\right)$ are constructed using the Gram-Schmidt process applied to $\left(Z_{1}, Z_{2}, e_{3}, \cdots, e_{m+2}\right)$. Denote by $P$ the frame change matrix field from $\left(e_{1}, \cdots, e_{m+2}\right)$ to $\left(Z_{1}, Z_{2}, N_{1}, \cdots, N_{m}\right)$.

3. Embed $I_{0}$ into the canonical frame $\left(e_{1}, \cdots, e_{m+2}\right):\left(I_{0}^{1}, \cdots, I_{0}^{m}\right) \longrightarrow\left(0,0, I_{0}^{1}, \cdots, I_{0}^{m}\right)$.

4. Compute the components of $I_{0}$ in the frame $\left(Z_{1}, Z_{2}, N_{1}, \cdots, N_{m}\right)$ : $\left(J_{0}^{1}, \cdots, J_{0}^{m+2}\right)^{T}:=P^{-1}\left(0,0, I_{0}^{1}, \cdots, I_{0}^{m}\right)^{T}$.

5. Perform the algorithm (4.8) on $\left(J_{0}^{1}, \cdots, J_{0}^{m+2}\right)^{T}$ with stopping criteria

$$
\left|\left\|\xi^{n+1}\right\|_{L_{2}}-\left\|\xi^{n}\right\|_{L_{2}}\right|<0.1
$$


TABLE 4.1

Increase of PSNR for different moving frames and parameters $\mu$ tested on the grey-level image "Kodim03" for different noise levels. From top to bottom: $\sigma=5,15,25$.

\begin{tabular}{|c|c|c|c|c|c|c|}
\hline Parameter $\mu$ & 1 & 0.1 & 0.01 & 0.007 & 0.001 & 0 \\
\hline Metric frame & -2.11 & -0.04 & 4.13 & $\mathbf{4 . 3 2}$ & 3.40 & 3.29 \\
\hline Previous approach & -2.07 & -0.02 & 4.13 & $\mathbf{4 . 3 2}$ & 3.40 & 3.29 \\
\hline Random frame & -1.88 & 0.01 & 4.09 & 4.30 & 3.41 & 3.29 \\
\hline Parameter $\mu$ & 1 & 0.1 & 0.01 & 0.004 & 0.001 & 0 \\
\hline Moving frame & -1.97 & -0.80 & 6.22 & $\mathbf{8 . 1 9}$ & 7.76 & 7.64 \\
\hline Metric frame & -1.74 & -0.73 & 6.21 & 8.18 & 7.76 & 7.64 \\
\hline Previous approach & -1.46 & -0.57 & 6.19 & 8.16 & 7.76 & 7.64 \\
\hline Random frame & 1 & 0.1 & 0.01 & 0.003 & 0.001 & 0 \\
\hline Moving frame Parameter $\mu$ & -1.65 & -0.90 & 6.72 & $\mathbf{1 0 . 4 7}$ & 9.96 & 9.75 \\
\hline Metric frame & -1.36 & -0.76 & 6.71 & 10.46 & 9.96 & 9.75 \\
\hline Previous approach & -1.13 & -0.61 & 6.74 & 10.46 & 9.96 & 9.75 \\
\hline Random frame & & & &
\end{tabular}

where the scalar $\lambda$ in (4.9) is considered as a Lagrange multiplier, updated at each iteration by

$$
\lambda=\frac{\sqrt{m|\Omega|} \sigma}{\left\|\nabla^{*} \xi^{n}\right\|_{L_{2}}}
$$

as suggested in [14] in the scalar case.

As it is usually done in the scalar case, the operators $\nabla$ and its adjoint $\nabla^{*}$ in (4.8) are respectively computed using forward and backward differences.

6 . Compute the components of the result $J$ in the canonical frame $\left(e_{1}, \cdots, e_{m+2}\right)$ : $\left(I^{1}, \cdots, I^{m+2}\right)^{T}=P\left(J^{1}, \cdots, J^{m+2}\right)^{T}$, and return the function $\left(I^{3}, \cdots, I^{m+2}\right)$.

4.5.1. Selection of parameters. In this Section, we test our denoising method for several values of $\mu$ and three different orthonormal moving frames: the two ones detailed in Sect. 4.4, as well as a moving frame where $Z_{1}$ is chosen randomly in the tangent bundle, and $Z_{2}$ is its unique orthonormal (up to a sign) in the tangent bundle. As for the two others moving frames, the vector fields $N_{1}, \cdots, N_{m}$ completing the moving frame are then constructed from the Gram-Schmidt process applied to $\left(Z_{1}, Z_{2}, e_{3}, \cdots, e_{m+2}\right)$. As well as the standard PSNR measure, we evaluate our method by computing the Q-Index [45], an image quality measure having higher perceptual correlation than PSNR and SNR-based metrics [37].

From Table 4.1 to Table 4.4, we report the increases of PSNR and Q-Index when testing our method on the grey-level and color image "kodim03" of the Kodak database for the noise levels $\sigma=5,15,25$ and different values of $\mu$ : 1,0.1,0.01,0.001,0. We also provide the best results for each moving frame (the "optimal" parameters $\mu$ were found empirically). The tables show that the "optimal" values of $\mu$ are the same for all the moving frames and are always in the range [0.001,0.01]. Moreover, we observe that the results are very bad when $\mu$ gets too high, and the results are similar for all the moving frames when $\mu$ is small enough. The best results are always obtained when the moving frame we deal with is the metric frame but in a very small 
TABLE 4.2

Increase of $Q$-Index (in \%) for different moving frames and parameters $\mu$ tested on the grey-level image "Kodim03" for different noise levels. From top to bottom: $\sigma=5,15,25$.

\begin{tabular}{|c|c|c|c|c|c|c|}
\hline Parameter $\mu$ & 1 & 0.1 & 0.01 & 0.006 & 0.001 & 0 \\
\hline Moving frame & 0.16 & 4.53 & 23.05 & $\mathbf{2 5 . 2 0}$ & 21.29 & 20.77 \\
\hline Metric frame & 0.84 & 4.59 & 23.09 & $\mathbf{2 5 . 2 0}$ & 21.28 & 20.77 \\
\hline Previous approach & 1.52 & 4.86 & 22.78 & 25.12 & 21.34 & 20.77 \\
\hline Random frame & 1 & 0.1 & 0.01 & 0.004 & 0.001 & 0 \\
\hline Moving frame & 3.34 & 8.05 & 49.50 & $\mathbf{6 8 . 8 2}$ & 61.67 & 59.38 \\
\hline Metric frame & 5.22 & 9.30 & 49.25 & 68.70 & 61.67 & 59.38 \\
\hline Previous approach & 6.92 & 9.88 & 49.01 & 68.53 & 61.73 & 59.38 \\
\hline Random frame & 1 & 0.1 & 0.01 & 0.003 & 0.001 & 0 \\
\hline Moving frame Parameter $\mu$ & 7.42 & 12.59 & 70.35 & $\mathbf{1 1 2 . 5 0}$ & 95.17 & 90.03 \\
\hline Metric frame & 10.25 & 15.37 & 70.23 & 112.40 & 95.17 & 90.03 \\
\hline Previous approach & 12.59 & 15.93 & 70.45 & 112.44 & 95.21 & 90.03 \\
\hline Random frame & & & &
\end{tabular}

TABLE 4.3

Increase of PSNR for different moving frames and parameters $\mu$ tested on the color image "Kodim03" for different noise levels. From top to bottom: $\sigma=5,15,25$.

\begin{tabular}{|c|c|c|c|c|c|c|}
\hline Parameter $\mu$ & 1 & 0.1 & 0.01 & 0.005 & 0.001 & 0 \\
\hline Moving frame & -2.49 & -0.56 & 4.55 & $\mathbf{5 . 0 8}$ & 4.27 & 4.12 \\
\hline Metric frame & -2.45 & -0.46 & 4.56 & 5.05 & 4.28 & 4.12 \\
\hline Previous approach & -2.47 & -0.52 & 4.47 & 5.06 & 4.29 & 4.12 \\
\hline Random frame & 1 & 0.1 & 0.01 & 0.004 & 0.001 & 0 \\
\hline Moving frame & -2.17 & -0.98 & 6.53 & $\mathbf{8 . 9 9}$ & 8.41 & 8.24 \\
\hline Metric frame & -2.10 & -0.85 & 6.59 & 8.96 & 8.40 & 8.24 \\
\hline Previous approach & -2.13 & -0.90 & 6.51 & 8.94 & 8.41 & 8.24 \\
\hline Random frame & 1 & 0.1 & 0.01 & 0.003 & 0.001 & 0 \\
\hline Moving frame Parameter $\mu$ & -1.75 & -0.85 & 6.90 & $\mathbf{1 1 . 1 1}$ & 9.54 & 9.46 \\
\hline Metric frame & -1.66 & -0.72 & 6.98 & 11.10 & 10.36 & 9.46 \\
\hline Previous approach & -1.71 & -0.78 & 6.94 & 11.10 & 9.54 & 9.46 \\
\hline Random frame & & & &
\end{tabular}

extent since the corresponding $\mu$ has a small value. Finally, "the optimal" $\mu$ tend to decrease when the noise level increases.

For the sake of shortness, we only provide the results on a single image, but we would like to point out that we observe the same properties when testing our method on other images.

We then report on Table 4.5 and Table 4.6 the means over the whole database of both increases of PSNR and Q-Index for our method tested with the metric frame and the range of values $[0.001,0.01]$ for the parameter $\mu$. Indeed, there is no need to 
TABLE 4.4

Increase of Q-Index (in \%) for different moving frames and parameters $\mu$ tested on the color image "Kodim03" for different noise levels. From top to bottom: $\sigma=5,15,25$.

\begin{tabular}{|c|c|c|c|c|c|c|}
\hline Parameter $\mu$ & 1 & 0.1 & 0.01 & 0.007 & 0.001 & 0 \\
\hline Moving frame & -4.13 & 1.76 & 30.46 & $\mathbf{3 2 . 6 3}$ & 23.13 & 22.07 \\
\hline Metric frame & -3.98 & 2.55 & 30.48 & 32.50 & 23.17 & 22.07 \\
\hline Previous approach & -4.06 & 2.21 & 30.04 & 32.49 & 23.39 & 22.07 \\
\hline Random frame & 1 & 0.1 & 0.01 & 0.004 & 0.001 & 0 \\
\hline Poving frame & -1.77 & 3.56 & 61.38 & $\mathbf{8 7 . 4 8}$ & 67.23 & 62.99 \\
\hline Metric frame & -1.25 & 4.88 & 61.53 & 87.08 & 67.22 & 62.99 \\
\hline Previous approach & -1.86 & 4.06 & 60.99 & 87.07 & 67.38 & 62.99 \\
\hline Random frame & 1 & 0.1 & 0.01 & 0.004 & 0.001 & 0 \\
\hline Parameter $\mu$ & 2.14 & 8.45 & 80.81 & $\mathbf{1 3 1 . 0 8}$ & 96.06 & 94.03 \\
\hline Moving frame & 2.97 & 10.07 & 81.05 & 130.63 & 89.41 & 94.03 \\
\hline Metric frame & 2.00 & 8.77 & 80.55 & 130.41 & 96.08 & 94.03 \\
\hline Previous approach & & & &
\end{tabular}

test the three moving frames since the results are similar according to the previous experiment, neither to test higher or lower values of $\mu$ since $[0.001,0.01]$ is the range of "optimal" values. The results confirm that the "optimal" $\mu$ tend to decrease when the noise level increases.

At last, Fig. 4.3 shows the output image of our algorithm tested on the color image "Kodim03" with the metric frame and $\sigma=25$ for different values of $\mu$. We test the values $\mu=0.1, \mu=0.004$ which is the value that provides the best result in terms of Q-Index, and $\mu=0$ which corresponds to the result of Bresson and Chan's method as mentioned in Sect. 4.4. In the previous Section, we were pointing out that $\mu$ can be viewed as a smoothing parameter for the moving frame. By observing the three images, it turns out that $\mu$ can also be viewed as a smoothing parameter for the output of our denoising method. More precisely, high values of $\mu$ make the result preserve the details of the original noisy image including the noise, whereas small values of $\mu$ remove all the noise but tend to produce over-smoothed results. The fact that $\mu$ is actually a smoothing parameter for the output image explains why the values of $\mu$ that provide the best results tend to decrease when the noise level increases as reported in the Tables.

We claim that our method is independent to the chosen orthonormal frame field $\left(Z_{1}, Z_{2}, N_{1}, \cdots, N_{m}\right)$, provided that $Z_{1}, Z_{2}$ are tangent vector fields to the surface $S$, in the sense that our best results are almost equivalent for the three moving frames tested, even for the one where the orthonormal moving frame $\left(Z_{1}, Z_{2}\right)$ of $T S$ is chosen randomly.

Even if we are not able to prove theoretically this invariance, we intuit that this is due to the fact that the best results are obtained for very small values of $\mu$. Indeed, assuming that $Z_{1}, Z_{2}$ are tangent vector fields to the surface $S$, the moving frame $\left(Z_{1}, Z_{2}, N_{1}, \cdots, N_{m}\right)$ tends to the original frame $\left(e_{1}, e_{2}, \cdots, e_{m+2}\right)$ when $\mu$ tends to 0 and consequently the components $\left(J_{0}^{1}, \cdots, J_{0}^{m+2}\right)$ of the original noisy im- 
TABLE 4.5

Average increase of PSNR (top) and Q-Index (in \%) (bottom) over the Kodak database for our method tested with the metric frame for different values of $\mu$ and noise levels $\sigma$ : the grey case.

\begin{tabular}{|c|c|c|c|c|c|c|c|c|c|c|c|c|}
\hline$\sigma^{\mu}$ & 0.01 & 0.009 & 0.008 & 0.007 & 0.006 & 0.005 & 0.004 & \multicolumn{2}{|c|}{0.003} & 0.002 & \multicolumn{2}{|c|}{0.001} \\
\hline 5 & 2.22 & 2.25 & 2.25 & 2.24 & 2.20 & 2.11 & 1.98 & \multicolumn{2}{|c|}{1.79} & 1.5 & \multicolumn{2}{|c|}{1.37} \\
\hline 15 & 4.48 & 4.69 & 4.90 & 5.08 & 5.23 & 5.33 & 5.32 & \multicolumn{2}{|c|}{5.12} & 4.9 & \multicolumn{2}{|c|}{4.67} \\
\hline 25 & 5.30 & 5.65 & 6.01 & 6.38 & 6.72 & 7.00 & 7.12 & \multicolumn{2}{|c|}{7.08} & 6.8 & \multicolumn{2}{|c|}{6.53} \\
\hline & 0.01 & 0.009 & 0.008 & 0.007 & 0.006 & 0.0 & 0. & & & & 0.002 & 0.001 \\
\hline$\sigma=5$ & 10 & 10.30 & 10.60 & 10.87 & 11.08 & 11. & & & & & 10.00 & 9.30 \\
\hline$\sigma=15$ & 29.25 & 30.33 & 31.42 & 32.49 & 33.46 & 34. & & & & & 29.37 & 27.66 \\
\hline$\sigma=25$ & 48.14 & 50.30 & 52.60 & 54.68 & 56.71 & 58. & & & & & 48.60 & 43.90 \\
\hline
\end{tabular}

TABLE 4.6

Average increase of PSNR (top) and Q-Index (in \%) (bottom) over the Kodak database for our method tested with the metric frame for different values of $\mu$ and noise levels $\sigma$ : the color case.

\begin{tabular}{|c|c|c|c|c|c|c|c|c|c|c|}
\hline \multicolumn{1}{|c|}{$\mu$} & 0.01 & 0.009 & 0.008 & 0.007 & 0.006 & 0.005 & 0.004 & 0.003 & 0.002 & 0.001 \\
\hline$\sigma=5$ & 2.67 & 2.77 & 2.86 & 2.93 & $\mathbf{2 . 9 7}$ & $\mathbf{2 . 9 7}$ & 2.90 & 2.74 & 2.51 & 2.26 \\
\hline$\sigma=15$ & 4.80 & 5.11 & 5.42 & 5.72 & 6.01 & 6.23 & $\mathbf{6 . 3 5}$ & 6.29 & 6.06 & 5.76 \\
\hline$\sigma=25$ & 5.44 & 5.88 & 6.35 & 6.84 & 7.32 & 7.74 & $\mathbf{8 . 0 2}$ & $\mathbf{8 . 0 2}$ & 7.72 & 7.31 \\
\hline \hline$\mu$ & 0.01 & 0.009 & 0.008 & 0.007 & 0.006 & 0.005 & 0.004 & $0 . .003$ & 0.002 & 0.001 \\
\hline$\sigma=5$ & 12.37 & 12.67 & 12.89 & $\mathbf{1 2 . 9 5}$ & 12.78 & 12.23 & 11.18 & 9.63 & 7.87 & 6.36 \\
\hline$\sigma=15$ & 36.98 & 38.89 & 40.82 & 42.69 & 44.28 & $\mathbf{4 5 . 1 8}$ & 44.52 & 41.31 & 35.83 & 30.28 \\
\hline$\sigma=25$ & 56.03 & 59.55 & 63.20 & 66.87 & 70.21 & $\mathbf{7 2 . 4 7}$ & 71.85 & 66.27 & 55.92 & 45.33 \\
\hline
\end{tabular}

age $\left(0,0, I_{0}^{1}, \cdots, I_{0}^{m}\right)$ in the moving frame tend to the image itself. As a consequence, for small values of $\mu$, the frames and the components in the frames are close to each other making the results be close to each others. Note that in the case of a grey-level image, the uniqueness of the unit normal to the surface $S$ make the components $J_{3}$ of the image in the different moving frames be the same for any value of $\mu$.

Preliminary tests that we do not present in this paper show that we obtain very bad results when the whole orthonormal moving frame is chosen randomly. We can then deduce that it is crucial that the moving frame we deal with encodes the local geometry of the image. In this paper, the moving frames encoding the local geometry of the image are satisfying the decomposition $T S \oplus N$, where $T S$ is the tangent bundle of $S$ and $N$ the normal bundle to $S$, but we would like to point out that other choices, that we do not discuss in this paper, are possible.

4.5.2. Comparison with state-of-the-art methods. As our denoising method belongs to the category of local variational methods, we compare our results with state-of-the-art denoising methods in the same category. More precisely, we compute and compare the means over the whole database of both increases of PSNR and QIndex between the noisy and denoised images. The parameters of the methods we compare with do not have necessarily the same nature as the ones of our method, but 


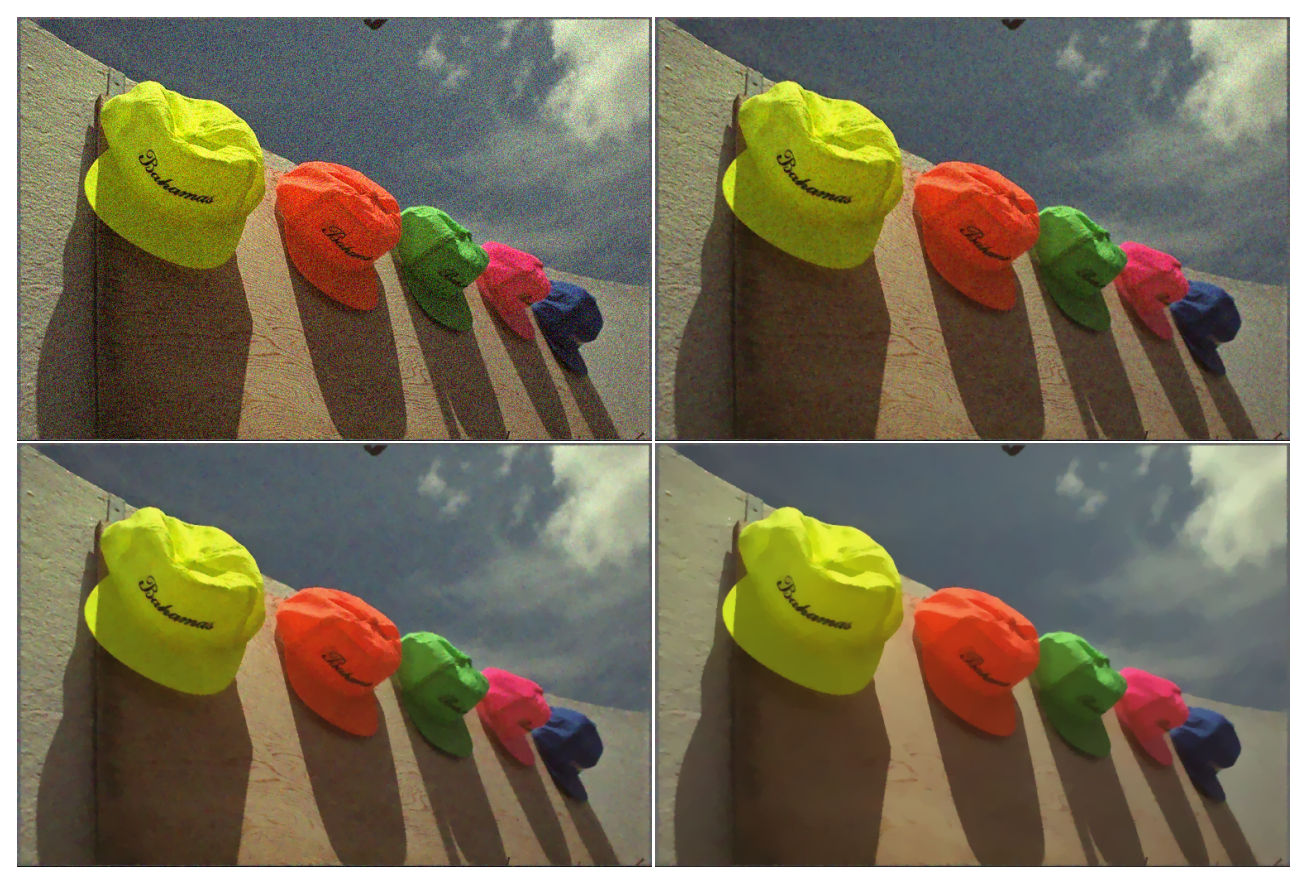

FIG. 4.3. Influence of the parameter $\mu$ in our denoising method. Clockwise from top-left: original noisy image, denoised image with $\mu=0.01$, denoised image with $\mu=0$, denoised image with $\mu=0.004$.

we tried to be as fair as possible in the comparisons by searching the values for the different parameters that provide the best results in each method.

Dealing with grey-level images, we compare our method with three variational denoising methods whose fidelity term, like our method, is not spatially adapted, namely the curvature-based denoising method of Bertalmío and Levine applied to the ROF model [9], Bregman iterations [36], and Chambolle's algorithm [14]. We also compare with the method of Gilboa et al. [23] that does contain a spatially adapted fidelity term dedicated to take into account the texture information of the image. Results for the noise levels $\sigma=5,15,25$ are available in Table 4.7 (increase of PSNR) and Table 4.8 (increase of Q-index).

The results for the curvature-based and Bregman iterations methods included in the tables have already been represented in [9], Figure 4.2. We obtained the results for Chambolle's algorithm using the demo available in IPOL [20]. We tested the approach of Gilboa et al. using the code provided at http://visl.technion.ac.il/ rilboa/PDE-filt/tv_denoising.html. The method is parametrized by the size of the window used for computing the local variance of the image. Modifying the size of the window has an effect on the smoothness of the output denoised image, meaning that it has a similar role as the scalar $\mu$ in our method. In the experiments, we keep the default parameter for the window size which is $5 \times 5$. We compare these results with our best results (according to Table 4.5).

We deduce from the results that our method performs well compared with similar approaches whose fidelity term does not involve spatial adaptivity. However, when the noise level increases, the results also show the limit of the methods that only 
TABLE 4.7

Comparison of different denoising methods. Average increase of PSNR over the Kodak database for different noise levels: the grey-level case.

\begin{tabular}{|c|c|c|c|}
\hline Noise level & $\sigma=5$ & $\sigma=15$ & $\sigma=25$ \\
\hline Denoising method & 1.28 & 4.77 & 6.80 \\
\hline Chambolle's algorithm (results from IPOL [20]) & 1.76 & 4.68 & 6.40 \\
\hline Bregman iterations (results from [9]) & $\mathbf{2 . 4 0}$ & 5.27 & 6.98 \\
\hline Curvature-based (results from [9]) & 2.25 & 5.33 & 7.12 \\
\hline VBTV (metric frame) & 2.01 & $\mathbf{5 . 4 8}$ & $\mathbf{7 . 2 8}$ \\
\hline Spatial adaptivity-based (code provided by the authors [23])
\end{tabular}

TABLE 4.8

Comparison of different denoising methods. Average increase of Q-Index (in \%) over the Kodak database for different noise levels: the grey-level case.

\begin{tabular}{|c|c|c|c|}
\hline Noise level & $\sigma=5$ & $\sigma=15$ & $\sigma=25$ \\
\hline Denoising method & 9.43 & 28.90 & 49.93 \\
\hline Chambolle's algorithm (results from IPOL [20]) & 11.32 & 31.35 & 50.17 \\
\hline Bregman iterations (results from [9]) & 10.79 & 35.02 & 60.43 \\
\hline Curvature-based (results from [9]) & 11.19 & 34.51 & 58.39 \\
\hline VBTV (metric frame) & $\mathbf{1 1 . 6 0}$ & $\mathbf{3 9 . 6 2}$ & $\mathbf{6 4 . 6 8}$ \\
\hline Spatial adaptivity-based (code provided by the authors [23])
\end{tabular}

involve geometric information (gradient, curvature), compared to the methods that involve features of higher level like texture for the method of Gilboa et al. or patches for the non local methods (see e.g. [13]).

Dealing with color images, we compare our best results (according to Table 4.6) with two denoising methods that use vectorial extensions of the Total Variation: the Vectorial Total Variation of Bresson and Chan [11] using the demo available in IPOL [20], as well as the Geometric Total Variation of Goldluecke et al. [25] whose code is available at www. sourceforge.net/p/cocolib. We also compare our method with a color extension of the Split Bregman algorithm [26] using the demo available in IPOL [22], and we test an extension of the spatially adapted method of Gilboa et al. [23] by applying it on each component of the color images. Note that the curvature-based denoising method in [9] that we compared with in the case of grey-level images has not been designed, as the method of Gilboa et al., for multi-channel images. Results are available in Table 4.9 (increase of PSNR) and Table 4.10 (increase of Q-Index).

The method of Goldluecke et al. [25] is parametrized by a scalar $\beta$ which is used as a smoothing parameter and the number of iterations of the iterative scheme to reach the solution. Fixing the number of iterations at 100 (which seems to be reasonable according to the tests performed in [25]), we tested several values for $\beta$ in order to provide the best results in terms of PSNR and Q-index. The optimal values we get are $\beta=0.02,0.06,0.12$ corresponding respectively to $\sigma=5,15,25$ for the increase of PSNR and $\beta=0.02,0.06,0.11$ corresponding respectively to $\sigma=5,15,25$ for the increase of Q-Index. As in the grey-level case, we test the spatially adapted denoising method of Gilboa et al. with the default parameter for the window size.

We observe that our method outperforms the other methods in both PSNR and 
TABLE 4.9

Comparison of different denoising methods. Average increase of PSNR over the Kodak database for different noise levels: the color case.

\begin{tabular}{|c|c|c|c|}
\hline Noise level & $\sigma=5$ & $\sigma=15$ & $\sigma=25$ \\
\hline Denoising method & 1.52 & 5.73 & 7.65 \\
\hline Split Bregman (results from IPOL [22]) & 2.11 & 5.73 & 7.58 \\
\hline Vectorial Total Variation (results from IPOL [20]) & 2.34 & 5.85 & 7.82 \\
\hline Geometric Total Variation (code provided by the authors [25]) & $\mathbf{6 . 3 5}$ & $\mathbf{8 . 0 2}$ \\
\hline VBTV (metric frame) & 2.34 & 6.06 & 7.79 \\
\hline Spatial adaptivity-based (code provided by the authors [23])
\end{tabular}

TABLE 4.10

Comparison of different denoising methods. Average increase of Q-Index (in \%) over the Kodak database for different noise levels: the color case.

\begin{tabular}{|c|c|c|c|}
\hline Noise level & $\sigma=5$ & $\sigma=15$ & $\sigma=25$ \\
\hline Denoising method & 2.59 & 31.36 & 54.61 \\
\hline Split Bregman (results from IPOL [22]) & 8.27 & 34.08 & 54.36 \\
\hline Vectorial Total Variation (results from IPOL [20]) & 10.94 & 41.65 & 72.34 \\
\hline Geometric Total Variation (code provided by the authors [25]) & $\mathbf{1 2 . 9 5}$ & $\mathbf{4 5 . 1 8}$ & $\mathbf{7 2 . 4 7}$ \\
\hline VBTV (metric frame) & 8.32 & 38.61 & 59.85 \\
\hline Spatial adaptivity-based (code provided by the authors [23])
\end{tabular}

Q-Index measures for any noise level. As expected, the channel-wise extension of the spatially adapted denoising method does not provide as good results as it does in the grey-level case.

On Fig. 4.4, we compare visually our denoising method with two of the four methods listed in Tables 4.9 and 4.10, namely Bresson and Chan's method, and the channel-wise extension of Gilboa et al.'s method. We do not compare with the method of Goldluecke et al. on purpose since we do not use the same noisy images (their algorithm automatically generates a noisy image from the input clean image). We compare the methods on a homogeneous region (top row), and regions containing both contours and textures (middle and bottom row). Whereas Bresson and Chan's method tends to provide over-smoothed results, both our method and the method based on spatial adaptivity preserve quite well the contours and textures of the original clean images. However, the spatial adaptivity-based method tends to create artifacts.

Our method does not smooth enough on the homogeneous region because the value of the smoothing parameter $\mu$ is too high. On the other hand, decrease $\mu$ will produce an over-smoothed result that tends to the result of Bresson and Chan when $\mu$ tends to 0 . Hence, in order to improve our method, we need at some point to incorporate higher level information like texture at it is done in Gilboa et al.

5. Conclusion and further work. We have introduced a generalization of the gradient operator to a vector bundle, from which we derived a generalization of the Rudin-Osher-Fatemi (ROF) denoising model by extending the Total Variation to a vector bundle. Assuming that the vector bundle is equipped with a positive definite metric and a covariant derivative compatible with the metric, we showed that Chambolle's algorithm for reaching the solution of the original ROF model can be 

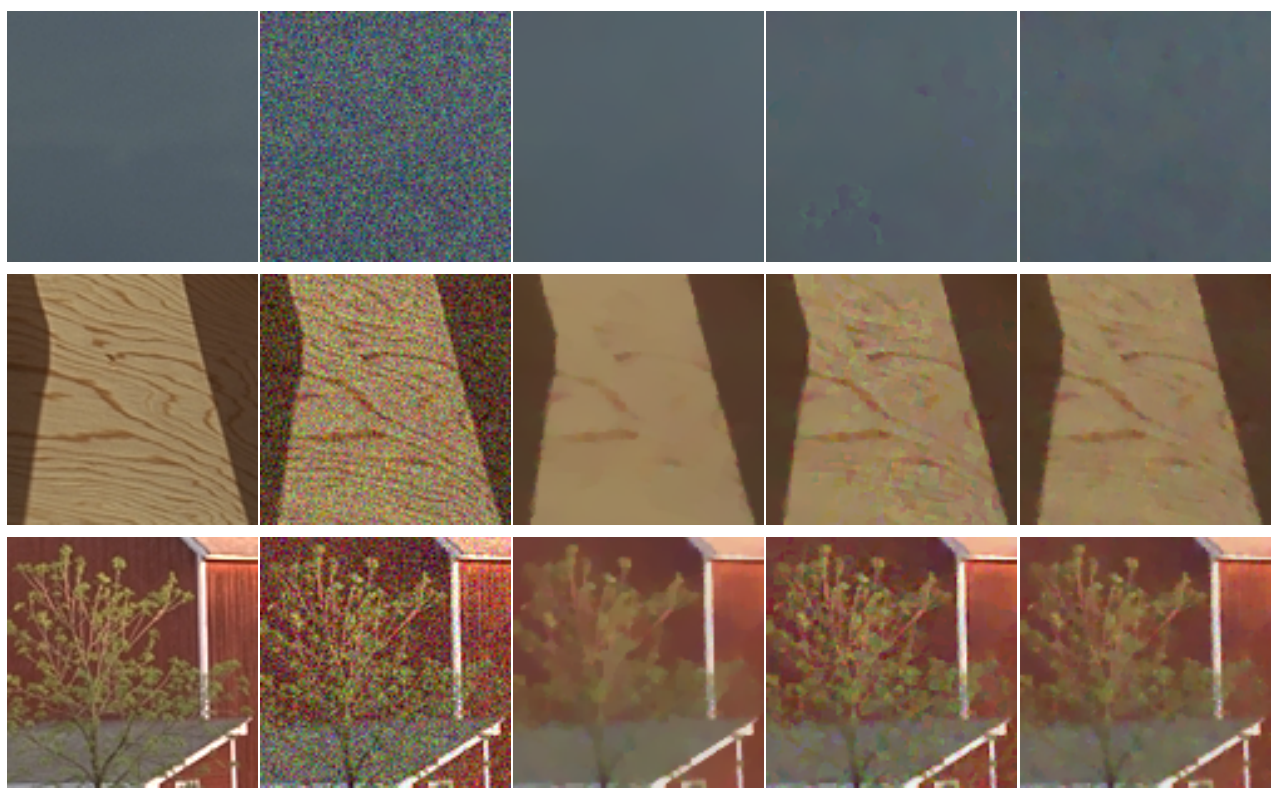

FIG. 4.4. Comparison of different denoising methods. From left to right: original patch, original patch corrupted with additive Gaussian noise of standard deviation 25, result of Bresson and Chan's method, result of the component-wise extension of Gilboa et al.'s method, result of our method tested with the metric frame.

extended to our denoising model.

For some particular covariant derivatives, we showed that our denoising model was equivalent to applying Bresson and Chan's [11] denoising model on the components of the noisy image in some orthonormal moving frames. Experiments showed that this approach outperforms state-of-the-art local denoising methods for color images.

We are currently investigating two non-local extensions of the denoising model proposed in this paper.

1. We are constructing a non-local gradient operator on a vector bundle following the way Gilboa and Osher [24] extended the Euclidean gradient operator to a non-local operator.

2. In [7],[8] and in the current paper, the denoising techniques that were applied to the components of the noisy image in some orthonormal moving frames were local methods. However, it turns out that other denoising techniques can be applied to these components, including the non-local ones.

Acknowledgements. The authors thank the anonymous reviewers for helpful remarks and suggestions.

\section{REFERENCES}

[1] L. AMBRosio, N. FUSCO, AND D. PALlARA, Functions of Bounded Variation and Free Discontinuity Problems, Oxford University Press, 2000.

[2] S. P. AWATE AND R. T. WHITAKER, Unsupervised, information-theoretic, adaptive image filtering for image restoration, IEEE Trans. Pattern Anal. Mach. Intell., 28(3) (2006), pp. 364-376.

[3] T. BATARD, M. BERTHIER, AND C. SAINT-JEAN, A metric approach to nD images edge detection with Clifford algebras, J. Math Imaging Vision, 33(3) (2009), pp. 296-312. 
[4] T. BATARD, Clifford bundles: a common framework for images, vector fields and orthonormal frame fields regularization, SIAM J. Imaging Sci., 3(3) 2010, pp. 670-701.

[5] T. BATARD, Heat equations on vector bundles - application to color image regularization, J. Math Imaging Vision, 41(1-2) (2011), pp. 59-85.

[6] T. BATARD AND N. SOCHEN, A class of generalized Laplacians devoted to multi-channel image processing, J. Math Imaging Vision, 48(3) (2014), pp. 517-543.

[7] T. BATARD AND M. BERTHIER, Spinor Fourier transform for image processing, IEEE J. Selected Topics Signal Process. (Special Issue on Differential Geometry in Signal Processing), 7(4) (2013), pp. 605-613.

[8] T. BATARD AND M. BERTALMÍO, Generalized gradient on vector bundle - application to image denoising, Proc. 4th Int. Conf. Scale-Space and Variational Methods in Computer Vision, A. Kuijper et al. Eds, LNCS 7893, 2013, pp. 12-23.

[9] M. BERTALMÍO AND S. LEVINE, Denoising an image by denoising its curvature image, SIAM J. Imaging Sci., 7(1) (2014), pp. 187-211.

[10] K. BREDIES, K. KUNISCH, AND T. POCK, Total generalized variation, SIAM J. Imaging Sci., 3(3) (2010), pp. 492-526.

[11] X. BRESSON AND T. F. CHAN, Fast dual minimization of the vectorial total variation norm and applications to color image processing, Inverse Probl. Imaging, 2(4) (2008), pp. 455484 .

[12] T. BROX, J. WEICKERT, B. BURGETH, AND P. MRÁZEK, Nonlinear structure tensors, Image and Vision Computing, 24(2006), pp. 41-55.

[13] A. BUADES, B. COLL, AND J.-M. MOREL, A review of image denoising algorithms, with a new one, Multiscale Model. Simul., 4(2) (2005), pp. 490-530.

[14] A. CHAMBOLLE, An algorithm for total variation minimization and applications, J. Math. Imaging Vision, 20 (2004), pp. 89-97.

[15] A. Chambolle, V. CASElles, D. CREMERS, M. NOVAGA, AND T. POCK, An introduction to total variation for image analysis, Theoretical Foundations and Numerical Methods for Sparse Recovery, 9 (2010), pp. 263-340.

[16] S. DI ZENZO, A note on the gradient of a multi-image, Computer Vision, Graphics, and Image Processing, 33(1) (1986), pp. 116-125.

[17] R. DUITS AND E. FRANKEN: Left-invariant parabolic evolutions on $S E(2)$ and contour enhancement via invertible orientation scores part I: linear left-invariant diffusions on $S E(2)$, Quarterly of Applied Mathematics, AMS, 68(2) (2010), pp. 255-292.

[18] R. DUITS AND E. FRANKEN: Left-invariant parabolic evolutions on SE(2) and contour enhancement via invertible orientation scores Part II: nonlinear left-Invariant diffusions on invertible orientation scores, Quarterly of Applied Mathematics, AMS, 68(2) (2010), pp. 293-331.

[19] R. DUITS AND E. FRANKEN: Left-invariant diffusions on the space of positions and orientations and their application to crossing-preserving smoothing of HARDI images, Int. J. Comput. Vis, 92(3) (2011), pp. 231-264.

[20] J. DURAN, B. COLL, AND C. SBERT, Chambolle's projection algorithm for total variation denoising, Image Process. on Line (2013).

[21] T. GEORGIEV, Covariant derivatives and vision, Proc. 9th Eur. Conf. Computer Vision, A. Leonardis et al. Eds, LNCS 3954 (4), 2006, pp. 56-69.

[22] P. GETREUER, Rudin-Osher-Fatemi total variation denoising using split Bregman, Image Process. on Line (2012).

[23] G. GILBOA, Y.Y. ZEEVI, AND N. SOCHEN, Texture preserving variational denoising using an adaptive fidelity term, Proc. VLSM 2003.

[24] G. GILBOA AND S. OSHER, Nonlocal operators with applications to image processing, Multiscale Model. Simul., 7(3) (2008), pp. 1005-1028.

[25] B. GOLDLUECKE, E. STREKALOVSKIY, AND D. CREMERS, The natural vectorial total variation which arises from geometric measure theory, SIAM J. Imaging Sci., 5 (2012), pp. 537-563.

[26] T. GOLDSTEIN AND S. OSHER, The split Bregman method for L1 regularized problems, SIAM J. Imaging Sci., 2 (2009), pp. 323-343.

[27] M. GÖCKELER AND T. SCHÜCKER, Differential Geometry, Gauge Theories, and Gravity, Cambridge University Press (1989)

[28] Y. JIN, J. JOST, AND G. WANG, A new nonlocal $H^{1}$ model for image denoising, J. Math. Imaging Vision, 48(1) (2014), pp. 93-105.

[29] S. KOBAYASHI AND K. NOMIZU, Foundations of Differential Geometry, Wiley-Interscience (1996)

[30] J. J. KONDERAK, On sections of fiber bundles which are harmonic maps, Bull. Math. Soc. 
Sci. Math. Roumanie, (4) 90 (1999), pp. 341-352.

[31] H. B. LAWSON AND M.-L. MICHELSON, Spin Geometry, Princeton University Press (1989).

[32] F. LENZEN, F. BECKER, AND J. LELLMANN, Adaptive second-order total variation: an approach aware of slope discontinuities, Proc. 4th Int. Conf. Scale-Space and Variational Methods in Computer Vision, A. Kuijper et al. Eds, LNCS 7893, 2013, pp. 61-73.

[33] S. LEFKIMMIATIS, A. ROUSSOS, M. UNSER, AND P. MARAGOS, Convex generalizations of total variation based on the structure tensor with applications to inverse problems, Proc. 4th Int. Conf. Scale-Space and Variational Methods in Computer Vision, A. Kuijper et al. Eds, LNCS 7893, 2013, pp. 48-60.

[34] T. LINDEBERG, Scale-Space Theory in Computer Vision, Springer (1994).

[35] M. LYSAKER, S. OSHER, AND X.-C. TAI, Noise removal using smoothed normals and surface fitting, IEEE Trans. Image Process., 13(10) (2004), pp. 1345-1357.

[36] S. OSHER, M. BURGER, D. GOLDFARB, J. XU, AND W. YIN, An iterative regularization method for total variation-based image restoration, Multiscale Model. Simul., 4(2) (2005), pp. 460-489.

[37] M. PEDERSEN AND J. HARDEBERG, Full-reference image quality metrics: classification and evaluation, Found. Trends. Comp. Graphics and Vision, 7(1) (2011), pp. 1-80.

[38] G. ROSMAN, X.-C. TAI, L. DASCAL, AND R. KIMMEL, Polyakov action minimization for efficient color image processing, ECCV Workshops (1), LNCS 6554, 2010, pp. 50-61.

[39] G. ROSMAN, X.-C. TAI, R. KIMMEL, AND A.M. BRUCKSTEIN, Augmented-Lagrangian regularization of matrix-valued maps, Methods and Applications of Analysis, 21(1), (2014), pp. 121-138.

[40] A. ROUSSOS AND P. MARAGOS, Tensor-based Image diffusions derived from generalizations of the total variation and Beltrami functionals, Proc. IEEE Int. Conf. Image Process. (2010).

[41] L. I. RUDIN, S. OSHER, AND E. FATEMI, Nonlinear total variation based noise removal algorithms, Phys. D, 60 (1992), pp. 259-268.

[42] N. SOCHEN, R. KIMMEL, AND R. MALLADI, A general framework for low level vision, IEEE Trans. Image Process., 7(3) (1998), pp. 310-318.

[43] M. SPIVAK, A Comprehensive Introduction to Differential Geometry, Publish or Perish, 2nd edition (1990).

[44] D. TSCHUMPERLÉ AND R. DERICHE, Vector-valued image regularization with PDEs: a common framework for different applications, IEEE Trans. Pattern Anal. Mach. Intell., 27(4) (2005), pp. 506-517.

[45] Z. WANG AND A. BOVIK, A Universal Image Quality Index, IEEE Signal Process. Letters, 9(3) (2002), pp. 81-84.

[46] J. WEICKERT, Coherence-enhancing diffusion filtering, Int. J. Compu. Vision, 31(2/3) (1999), pp. 111-127.

[47] W. ZHU AND T. F. CHAN, Image denoising using mean curvature of image surface, SIAM J. Imaging Sci. 5(1) (2012), pp. 1-32. 